Supporting Information for:

\title{
Strong Magnetocrystalline Anisotropy Arising from Metal- Ligand Covalency in a Metal-Organic Candidate for 2D Magnetic Order
}

\author{
Yiran Wang, ${ }^{\dagger, \S}$ Michael E. Ziebel, ${ }^{\ddagger}, \S$ Lei Sun, ${ }^{\dagger}$ J. Tyler Gish, ${ }^{\uparrow}$ Tyler J. Pearson, ${ }^{\dagger}$ \\ Xue-Zeng Lu, ${ }^{\circledR}$ Agnes E. Thorarinsdottir, ${ }^{\dagger}$ Mark C. Hersam, ${ }^{\dagger, \uparrow}$ Jeffrey R. \\ Long, ${ }^{\dagger, \|,+*}$ Danna E. Freedman, ${ }^{\dagger,+, *}$ James M. Rondinelli, ${ }^{*, \pi}$ Danilo \\ Puggioni, ${ }^{*}$, and T. David Harris $*, \dagger, \$$ \\ tDepartment of Chemistry and Department of Materials Science and Engineering, \\ Northwestern University, Evanston, Illinois 60208, United States \\ Department of Chemistry and "Department of Chemical and Biomolecular Engineering, \\ University of California, Berkeley, California 94720, United States \\ ${ }^{\perp}$ Materials Sciences Division, Lawrence Berkeley National Laboratory, Berkeley, \\ California, 94720, United States \\ "Department of Chemistry, Massachusetts Institute of Technology, Cambridge, Massachusetts \\ 02139, United States
}

Email: dharris@berkeley.edu, danilo.puggioni@northwestern.edu, jrondinelli@northwestern.edu,danna@mit.edu,jrlong@,berkeley.edu

\section{Table of Contents}

Experimental Section $\quad$ S3

Magnetic Properties of Polycrystalline Samples Without Special Orientation $\quad$ S6

$\begin{array}{ll}\text { Difference in Magnetic Ordering Temperature for } 1 \text { and } 2 & \text { S7 }\end{array}$

$\begin{array}{ll}\text { Calculation of Magnetocrystalline Anisotropy Constants } & \text { S7 }\end{array}$

$\begin{array}{ll}\text { Table S1. Crystallographic data for } 2 & \text { S8 }\end{array}$

$\begin{array}{ll}\text { Figure S1. Thermogravimetric analysis data for } \mathbf{2} & \text { S9 }\end{array}$

Figure S2. Face indexing of a single crystal of $2 \quad$ S9

Figure S3. Experimental and simulated powder diffraction patterns of $2 \quad$ S10

Figure S4. Mössbauer spectra for $2 \quad$ S11

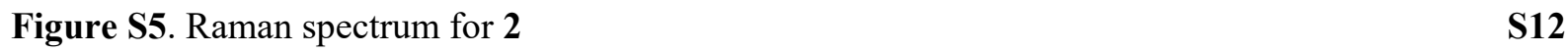

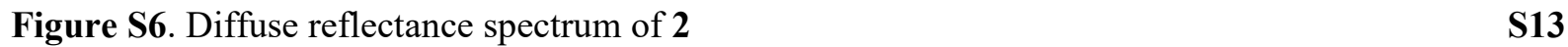

$\begin{array}{ll}\text { Figure S7. Electronic conductivity data for } 2 & \text { S14 }\end{array}$

Figure S8. Variable-temperature dc magnetic susceptibility data for $2 \quad$ S15

Figure S9. Variable-temperature $\chi_{\mathrm{M}} T$ vs $T$ plots for $\mathbf{2} \quad \mathbf{S 1 5}$

Figure S10. Variable-temperature ac magnetic susceptibility data for $2 \quad$ S16

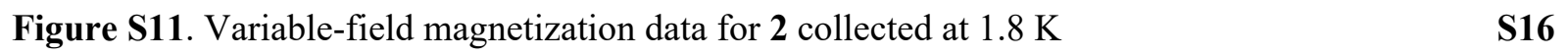

$\begin{array}{ll}\text { Figure S12. Variable-field magnetization data for } 2 \text { collected at } 10 \text { and } 20 \mathrm{~K} & \text { S17 }\end{array}$ 
$\begin{array}{ll}\text { Figure S13. Magnetic hysteresis data for } 2 & \text { S17 }\end{array}$

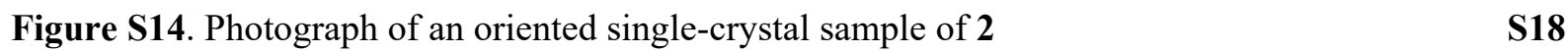

Figure S15. Variable-field magnetization data for an oriented single-crystal sample of $2 \quad$ S18

Figure S16. Plot of $\mathrm{d} M_{\mathrm{z}} / \mathrm{d} H$ vs $H$ for an oriented single-crystal sample of $\mathbf{2}$ S19

Figure S17. Magnetic hysteresis data for an oriented single-crystal sample of $2 \quad$ S20

Figure S18. Switching-field distribution plots for an oriented single-crystal sample of $2 \quad$ S21

Figure S19. Variable-field magnetic hysteresis data for oriented single-crystal sample of $2 \quad$ S22

Figure S20. Stoner-Wohlfarth fitting of oriented single-crystal data for $2 \quad$ S23

Figure S21. Sucksmith-Thompson fitting of oriented single-crystal data for $2 \quad$ S24

Figure S22. Spin density plots for DFT calculations, neglecting SOC $\quad$ S24

Figure S23. Charge density difference plots for DFT calculations, including SOC $\quad$ S25

$\begin{array}{ll}\text { Figure S24. DFT-calculated band structures for } \mathbf{2} & \text { S26 }\end{array}$

Figure S25. Comparison of DFT- and MLWF-calculated band structures $\quad$ S26

$\begin{array}{ll}\text { Figure S26. Molecular orbital model for a single iron center in } 2 & \text { S27 }\end{array}$

$\begin{array}{ll}\text { References } & \text { S28 }\end{array}$ 


\section{EXPERIMENTAL SECTION}

X-ray Structure Determination. A single crystal of $\mathbf{2}$ suitable for X-ray diffraction analysis was coated with deoxygenated Paratone-N oil and mounted on a MicroMounts rod. The crystallographic data were collected at $250 \mathrm{~K}$ on a Bruker APEX II diffractometer equipped with a $\mathrm{Cu} \mathrm{K} \alpha$ microsource. Raw data were integrated and corrected for Lorentz and polarization effects using Bruker APEX2 v. 2009.1, ${ }^{1}$ and absorption corrections were applied using SADABS. ${ }^{2}$ The space group assignment was determined by examination of systematic absences, E-statistics, and successive refinement of the structure. The structure was solved and refined with SHELXL ${ }^{3}$ operated with the OLEX2 interface. ${ }^{4}$ Solvent molecules were severely disordered and could not be modeled properly. Therefore, the solvent masking procedure as implemented in OLEX2 was used. A total of 90.2 electrons were estimated per unit-cell and ascribed to disordered DMF molecules. Due to this disorder and the general poor quality of the single-crystal X-ray diffraction data, the chemical formula is based on elemental analysis from $\mathrm{C}, \mathrm{H}$, and $\mathrm{N}$ combustion and thermogravimetric analysis (Figure S1). Hydrogen atoms for the tetramethylammonium ions were placed at calculated positions using suitable riding models and refined using isotropic displacement parameters derived from their parent atoms. Thermal parameters were refined anisotropically for all non-hydrogen atoms. Crystallographic data and the details of data collection are listed in Table S1.

Transmission Powder X-ray Diffraction Analysis. Powder X-ray diffraction (PXRD) data were collected at $298 \mathrm{~K}$ on a STOE-STADI-P powder diffractometer equipped with an asymmetric curved germanium monochromator $(\mathrm{Cu} \mathrm{K \alpha} 1$ radiation, $\lambda=1.54056 \AA$ ) and one-dimensional silicon strip detector (MYTHEN2 $1 \mathrm{~K}$ from DECTRIS). The line-focused $\mathrm{Cu}$ X-ray tube was operated at $40 \mathrm{kV}$ and $40 \mathrm{~mA}$. A solvated powder sample of 2 was sandwiched between two pieces of Kapton tape in the $3 \mathrm{~mm}$ hole of a metallic mask in a glovebox operated under a humid dinitrogen atmosphere. Intensity data from 5 to $30^{\circ} 2 \theta$ were collected over a period of $20 \mathrm{~min}$. The instrument was calibrated against a NIST silicon standard (640d) prior the measurement. These data are provided in Figure S3.

Raman Measurements. Crystals of $\mathbf{2}$ were deposited onto a silicon oxide-coated silicon wafer and sealed in a Linkam THMS350 V microscope state. Raman spectra were collected using a Horiba LabRam HR Evolution confocal microscope. Individual crystals were excited with a $473 \mathrm{~nm}$ continuous-wave diode laser (Cobalt Blue) at $250 \mu \mathrm{W}$ power equipped with a long working distance $50 \times$ microscope objective (NA $=0.50$; Nikon) and 1800 grooves $\mathrm{mm}^{-1}$ grating. Individual spectra were collected for $2 \mathrm{~min}$.

Reflection Powder X-ray Diffraction Analysis. PXRD data were collected at 298 K on a STOE-STADIMP powder diffractometer equipped with an asymmetric curved germanium monochromator $(\mathrm{Cu} \mathrm{K} \alpha 1$ radiation, $\lambda=1.54056 \AA$ ) and one-dimensional silicon strip detector (MYTHEN2 1K from DECTRIS). The line-focused $\mathrm{Cu}$ X-ray tube was operated at $40 \mathrm{kV}$ and $40 \mathrm{~mA}$. The sample was placed on a flat metallic holder and span during the measurement. Intensity data from 5 to $30^{\circ} 2 \theta$ were collected over a period of 30 min. The instrument was calibrated against a NIST silicon standard (640d) prior the measurement.

Inductively Coupled Plasma Optical Emission Spectroscopy. The oriented single-crystal sample was digested using concentrated $\mathrm{HNO}_{3}(0.5 \mathrm{~mL})$ and $\mathrm{H}_{2} \mathrm{O}_{2}$ solution $\left(30\right.$ wt. \% in $\left.\mathrm{H}_{2} \mathrm{O}, 0.1 \mathrm{~mL}\right)$ at $60{ }^{\circ} \mathrm{C}$ for 20 min. The resulting solution was filtered and diluted with $\mathrm{H}_{2} \mathrm{O}$ to a total volume of $8.576 \mathrm{~mL}$. The sample was analyzed using a Thermo S9 Scientific iCAP 7600 instrument. The radial optical emission signal was acquired and analyzed. A blank sample and a set of Fe standard solutions were measured immediately before measuring the sample. The average Fe concentration in the sample was $2.476 \mathrm{ppm}$. From the moles of $\mathrm{Fe}$ in the sample, the mass of the oriented single-crystal sample was calculated to be $0.264(8) \mathrm{mg}$.

Computational Details. First principles density-functional theory (DFT) plus Hubbard calculations $(U=$ $5 \mathrm{eV}$ ) including spin-orbit coupling (SOC) employed the Perdew-Burke-Ernzerhof exchange-correlation 
functional revised for solids (PBEsol) as implemented in the Vienna $a b$ initio simulation package (VASP) ${ }^{6,7}$ We used the projector augmented wave (PAW) approach to treat the core and valence electrons using the following valence configurations: $2 s^{2} 2 p^{4}(\mathrm{O}), 3 p^{6} 4 s^{2} 3 d^{6}(\mathrm{Fe}), 3 s^{2} 3 p^{5}(\mathrm{Cl})$, and $2 s^{2} 2 p^{2}(\mathrm{C}){ }^{8}$ An 8 $\times 8 \times 8 \Gamma$-centered $k$-point mesh was generated and a $500 \mathrm{eV}$ energy cutoff used for the planewave expansion. To treat van der Waals interactions, we used the DFT-D3 correction method. ${ }^{9}$ The experimental structure of $\mathbf{2}$ was used in all the calculations. For simplicity, cations were removed from the structure and a uniform background charge was used for the modeling of the dianionic unit cell.

After obtaining the DFT Bloch bands at the PBEsol $+U$ level, we constructed maximally localized Wannier functions (MLWFs) using the VASP2WANNIER90 interface and the wannier90 code. ${ }^{10,11}$ The spread of the MLWFs was considered to be converged if the change between two successive iterations of the spread minimization was smaller than $10^{-11}$. For the disentanglement procedure, we defined an outer energy window $[-23.4,0.8] \mathrm{eV}$ and an inner energy window of $[-23.4,0.7] \mathrm{eV}$. The MLWFs were obtained starting from an initial projection of atomic $p$ basis functions centered at the $\mathrm{C}$ and $\mathrm{O}$ sites within the unit cell onto the Bloch bands. The complex nature of the spinor Wannier functions makes their visualization not straightforward. Therefore, we plot the scalar Wannier functions, which result from the Wannierization of the spin-down channel neglecting SOC. This approach is justified by noting the similarity between the electronic structures with and without SOC. The spin density, charge density, and MLWF are plotted using an isolevel of $3.5 \times 10^{-5}, 3.5 \times 10^{-5}$, and $2.07 \times 10^{-5} \mathrm{e} / \AA^{3}$, respectively.

Exfoliation of 2 and Atomic Force Microscopy (AFM) Measurements. Framework material 2 was exfoliated in a $\mathrm{N}_{2}$ atmosphere glovebox $\left(\mathrm{O}_{2}\right.$ and $\mathrm{H}_{2} \mathrm{O}$ concentration $\left.<11 \mathrm{ppm}\right)$. The crystal was mechanically exfoliated using the Scotch tape method and deposited onto a $300 \mathrm{~nm} \mathrm{SiO} / 2 / \mathrm{Si}_{\text {substrate. The }}$ tapping mode AFM topography image was then taken immediately after exfoliation using an Asylum Cypher AFM system with a Si cantilever and a scanning rate of $1 \mathrm{~Hz}$.

Other Physical Measurements. Elemental analysis of $\mathbf{2}$ was performed by Midwest Microlab (Indianapolis, IN). Thermogravimetric analysis (TGA) was performed on a Netzsch Jupiter F3 STA coupled with gas chromatography-mass spectrometry (GC-MS) in a flow of helium at a heating rate of $10{ }^{\circ} \mathrm{C} \mathrm{min}{ }^{-1}$ between 30 and $600{ }^{\circ} \mathrm{C}$. Scanning Electron Microscopy (SEM) was performed on solvated crystals of 2. The crystals were sprinkled on a double-sided carbon tape which was fixed on a sample holder. Scanning electron imaging was carried out using Hitachi SU8030 SEM instrument. Zero-field iron-57 Mössbauer spectra for 2 were obtained at 4 and $80 \mathrm{~K}$ with a constant acceleration spectrometer and a cobalt- 57 rhodium source. Prior to the measurements, the spectrometer was calibrated at $295 \mathrm{~K}$ with $\alpha$-iron foil. Samples were prepared in a dinitrogen-filled glovebox, covered with deoxygenated Paratone-N oil, and frozen in liquid nitrogen prior to handling in air. The sample of 2 contained approximately $4 \mathrm{mg} \mathrm{cm}^{-2}$ of iron. The spectra were analyzed using the WMOSS Mössbauer Spectral Analysis Software (www.wmoss.org). 


\section{Magnetic Properties of Polycrystalline Samples Without Special Orientation}

To assess the magnetic behavior of $\mathbf{2}$, variable-temperature dc magnetic susceptibility data were collected. In the $\chi_{\mathrm{M}}$ vs $T$ plot (Figures 2 and S8), collected under a magnetic field of $10 \mathrm{Oe}$, the magnetization increases gradually when the temperature is decreased from $300 \mathrm{~K}$ and a sharp increase occurs around $40 \mathrm{~K}$, indicating the development of long-range magnetic order. Furthermore, the magnetic susceptibility data shows a divergence between data where the sample was cooled with or without an applied field below 40 $\mathrm{K}$, further supporting the onset of spontaneous magnetization. In addition, in the corresponding plot of $\chi_{\mathrm{M}} T$ vs $T$ (Figure S9), the value of $\chi_{\mathrm{M}} T$ at $300 \mathrm{~K}$ is $19.3 \mathrm{~cm}^{3} \mathrm{~K} \mathrm{~mol}^{-1}$ under an applied field of $10 \mathrm{Oe}$, which is larger than the value of $9.5 \mathrm{~cm}^{3} \mathrm{~K} \mathrm{~mol}^{-1}$ expected for two magnetically isolated paramagnetic $\mathrm{Fe}^{\mathrm{III}}$ centers and two radical ligands. This comparison indicates that some degree of short-range magnetic order is preserved between paramagnetic centers far above the ordering temperature $\left(T_{\mathrm{N}}\right)$.

To precisely determine the ordering temperature for $\mathbf{2}$, variable-temperature ac magnetic susceptibility measurements were conducted under zero applied dc field (Figure S10). The value of $\chi_{\mathrm{M}}{ }^{\prime \prime}$ deviates from zero at $34 \mathrm{~K}$, indicating the onset of long-range magnetic order. A slight frequency-dependence was observed for the peak in both the in-phase $\left(\chi_{\mathrm{M}}{ }^{\prime}\right)$ and the out-of-phase $\left(\chi_{\mathrm{M}}{ }^{\prime \prime}\right)$ magnetic susceptibility data for 2, giving a Mydosh parameter of $\varphi=0.074 .^{12}$ This frequency behavior is most consistent with a cluster spin-glass, in which interactions between strongly-coupled magnetic clusters lead to the formation of a glassy state. Such interactions could potentially emerge due to localization of the linker-centered spins at low temperature, although Raman data shows no electron localization down to at least $77 \mathrm{~K}$. Alternatively, antiferromagnetic interactions between the radical spins, which for a geometrically frustrated lattice, could explain the glassy behavior, although these interactions should be much weaker than the direct exchange interactions between iron centers and organic radicals. While the exact origin of the observed glassy behavior is not obvious from the experimental data presented here, we note that the low temperature magnetization data are consistent with a ferrimagnetic ground state rather than a spin glass ground state. Other ferrimagnetic materials, including several metal-organic materials containing radical linkers, display similar glassy behavior in ac susceptibility measurements, which has been attributed to structural disorder or competing ferromagnetic and antiferromagnetic interactions. ${ }^{13-14}$ We note that the glassy magnetic behavior observed in $\mathbf{1}$ and $\mathbf{2}$ is not present in the reduced analogue of $\mathbf{1},{ }^{13 \mathrm{c}}$ which may indicate that linker mixed-valence is important is explaining the glassy magnetic behavior.

To study the nature of coercivity in $\mathbf{2}$, variable-field dc magnetization data were collected. At $1.8 \mathrm{~K}$, the magnetization reaches saturation under an applied field of $70 \mathrm{kOe}$, giving a value of $M_{\mathrm{s}}=7.3 \mu_{\mathrm{B}}$ for $\mathbf{2}$. This value is close to the value of $8 \mu_{\mathrm{B}}$ which would be expected for an overall $S=4$ system, constituting the repeating unit of two $\mathrm{Fe}^{\mathrm{III}}$ ions antiferromagnetic coupled to two radical ligands (Figure S11-S12). The hysteresis loop remains open up to $20 \mathrm{~K}$ under a field-sweep rate of $0.08 \mathrm{Oe} \mathrm{s}^{-1}$. Moreover, coercive fields with values of $H_{\mathrm{ci}}=3013,114,14 \mathrm{Oe}$ at 20,10 and $1.8 \mathrm{~K}$, respectively, were obtained with a field-sweep rate of $1.94,0.71,0.08 \mathrm{Oe} \mathrm{s}^{-1}$, respectively (Figure $\mathrm{S} 13$ ). The hysteresis data collected at $1.8 \mathrm{~K}$ shows an unusual shape, with prominent steps, which is similar to the data reported for isostructural iron-quinoid frameworks, ${ }^{13 \mathrm{~b}, 13 \mathrm{c}}$ indicated a significant magnetocrystalline anisotropy in $\mathbf{2}$. Moreover, the coercive fields for the oriented single-crystal sample of $\mathbf{2}$ in two orthogonal directions (see main text for details) are larger than that of the polycrystalline sample, showing that the uniformity of the crystallite orientation has a significant influence on the coercive field. 


\section{Difference in Magnetic Ordering Temperature for 1 and 2}

Despite possessing spectroscopically similar electronic structures, we observe a large difference in the magnetic ordering temperatures of framework materials $1(80 \mathrm{~K})$ and $2(34 \mathrm{~K})$. The most apparent difference between these two framework materials in the much larger interlayer spacing of $\mathbf{2}$ compared to $\mathbf{1}$, which could suggest that dipole-dipole interactions between layers is critical for the stabilization of the magnetic ordering. We propose that, instead, the magnetic ordering temperature is much more dependent on the precise degree of metal-to-ligand electron transfer and possible electron localization at low temperatures.

The magnetic properties of a large number of layered $\mathrm{Fe}$-chloranilate frameworks have been reported over the last ten years. ${ }^{13}$ The local electronic structure of these frameworks is dependent on both the synthetic conditions and the charge-balancing cation. The Fe-chloranilate frameworks with no organic radical character or with less than one radical per three ligands consistently display magnetic ordering temperatures below $10 \mathrm{~K} .{ }^{13 a, d, g}$ In contrast, frameworks with at least two organic radicals per three ligands consistently display ordering temperatures above $20 \mathrm{~K} .{ }^{13 \mathrm{~b}, \mathrm{c}, \mathrm{f}, \mathrm{i}}$ Some of these frameworks deviate from a perfect honeycomb lattice, which favors localization of the organic radicals and reduces the magnetic ordering temperature ${ }^{13 \mathrm{f}}$ Further, desolvation of 1 suppresses the magnetic ordering temperature from $80 \mathrm{~K}$ to $26 \mathrm{~K}$ as a result of lattice distortion, though there are no clear spectroscopic indicators for a change in the electronic structure. ${ }^{13 \mathrm{~b}}$ Importantly, Fe-chloranilate frameworks with one radical spin per organic linker consistently display magnetic ordering temperatures above $100 \mathrm{~K}$, regardless of the interlayer distance, suggesting that in-plane magnetic ordering temperatures are the dominant factor in determining the ordering temperature of $\mathbf{1}$ and $\mathbf{2} .^{13 \mathrm{c}, \mathrm{h}, \mathrm{j}}$

Additionally, we note that the role of dipole-dipole interactions on magnetic ordering have been studied in other layered materials. For example, in layered double hydroxides, it has been shown that magnetic ordering temperature is minimally affected as the interlayer distance is expanded from 7.5 to $34 \AA{ }^{15}$ Consequently, interlayer dipole interactions are predominantly affected by in-plane magnetic interactions rather than interlayer distance. Specifically, near the in-plane magnetic ordering temperature, the magnetic correlation length greatly increases, which, in turn, increases the interlayer dipole-dipole interactions to induce 3D magnetic ordering. This process, in which in-plane ordering induces 3D magnetic ordering, has also been observed in other layered magnetic systems. ${ }^{16}$ As such, the lower magnetic ordering temperature of $\mathbf{2}$ relative to $\mathbf{1}$ can most likely be attributed to slight changes in the electronic structure compared to $\mathbf{1}$, rather than the increased interlayer distance.

\section{Calculation of Magnetocrystalline Anisotropy Constants}

The anisotropy constant $K_{\mathrm{u}}$ was calculated using the Stoner-Wohlfarth model, as described in the main text (Figure S20). Two linear fits were applied to the magnetization data with $H \perp c$ : One in the low-field regime and one in the high-field regime where the sample is saturated. The intersection of these two lines afforded the saturation magnetization as its ordinate and the saturation field as its abscissa. Because of imperfect crystal orientation, at temperatures above $1.8 \mathrm{~K}$, it was necessary to subtract a sharp increase in the magnetization data at low fields (which corresponds to a small percentage of crystals with $H \| c$ ). While this prevents absolute determination of anisotropy constants over the whole temperature range, the change in $K_{\mathrm{u}}$ relative to the value at $1.8 \mathrm{~K}$ could be reliably fit as a function of temperature.

As a secondary measure of magnetocrystalline anisotropy in $\mathbf{2}$, the anisotropy parameters $K_{1}$ and $K_{2}$ were calculated according to equation $\mathrm{S} 1$ :

$$
E_{a}=K_{1} \sin 2 \theta+K_{2} \sin 4 \theta+K_{3} \sin 6 \theta+\cdots
$$

where $K_{\mathrm{n}}$ is an anisotropy constant and $\theta$ is the angle between the axis of magnetization and the easy axis. For the uniaxial magnetocrystalline anisotropy of $\mathbf{2}$, only the first two terms are necessary. These anisotropy 
constants were calculated according to the Sucksmith-Thompson method. ${ }^{17}$ For a uniaxial crystalline material with an easy axis of magnetization, the following correlation holds:

$$
\frac{2 K_{1}}{M_{S}^{2}}+\frac{4 K_{2} M^{2}}{M_{S}^{4}}=\frac{H}{M}
$$

where $K_{1}$ and $K_{2}$ are the anisotropy constants defined in the main text, $M_{\mathrm{s}}$ is the saturation magnetization, $M$ is the experimentally observed magnetization, and $H$ is the applied magnetic field. Accordingly, we plotted $H / M_{\mathrm{z}}$ vs $M_{\mathrm{z}}{ }^{2}$, fitted the linear region of the plot with linear regression (Figure S21), and calculated $K_{1}$ and $K_{2}$ from the slope and intercept obtained from the fits, as well as the value of the saturation magnetization $\left(M_{\mathrm{s}}\right)$. Note that the nonlinear region at small values of $M_{\mathrm{z}}{ }^{2}$ is due to the low magnetization plateau, which corresponds to a random distribution of crystals with magnetization oriented parallel or antiparallel to the applied field. This method yielded values of $K_{1}=51 \mathrm{~kJ} / \mathrm{m}^{3}$ and $K_{2}=23 \mathrm{~kJ} / \mathrm{m}^{3}$. 
Table S1 | Crystallographic data for 2.

\begin{tabular}{|c|c|}
\hline & 2 \\
\hline Empirical formula & $\mathrm{C}_{47} \mathrm{H}_{73} \mathrm{Cl}_{6} \mathrm{Fe}_{2} \mathrm{~N}_{9} \mathrm{O}_{19}$ \\
\hline Formula wt $\left(\mathrm{g} \mathrm{mol}^{-1}\right)$ & 1392.5 \\
\hline Crystal system & trigonal \\
\hline Space group & $P \overline{3} 1 m$ \\
\hline Wavelength $(\AA)$ & 1.54178 \\
\hline Temperature (K) & 250 \\
\hline$a(\AA)$ & $13.5717(18)$ \\
\hline$b(\AA)$ & $13.5717(18)$ \\
\hline$c(\AA)$ & $9.9759(18)$ \\
\hline$\alpha\left(^{\circ}\right)$ & 90 \\
\hline$\beta\left(^{\circ}\right)$ & 90 \\
\hline$\gamma\left({ }^{\circ}\right)$ & 120 \\
\hline$V\left(\AA^{3}\right)$ & 1591.3(4) \\
\hline$Z$ & 3 \\
\hline$d_{\text {calc }}\left(\mathrm{g} \mathrm{cm}^{-3}\right)$ & 1.452 \\
\hline$\mu\left(\mathrm{mm}^{-1}\right)$ & 13.44 \\
\hline Reflns coll./unique & $2207 / 2180$ \\
\hline$R_{\text {int }}$ & 0.0705 \\
\hline$R_{1}(I>2 \sigma(I))^{a}$ & 0.01011 \\
\hline$w R_{2}(\text { all })^{b}$ & 0.2916 \\
\hline GoF & 1.090 \\
\hline
\end{tabular}




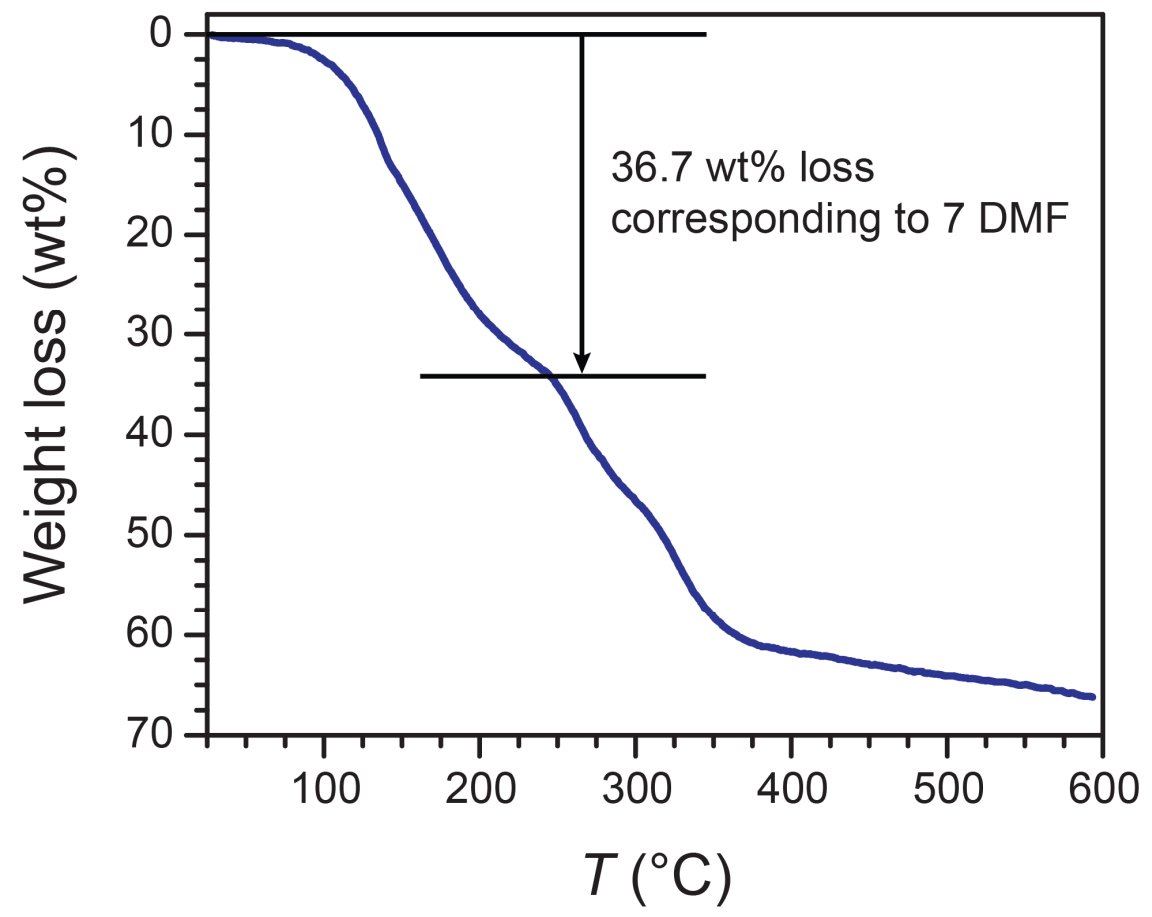

Figure S1. TGA data for 2, collected at a scan rate of $10{ }^{\circ} \mathrm{C} \mathrm{min}^{-1}$ under a flow of helium.
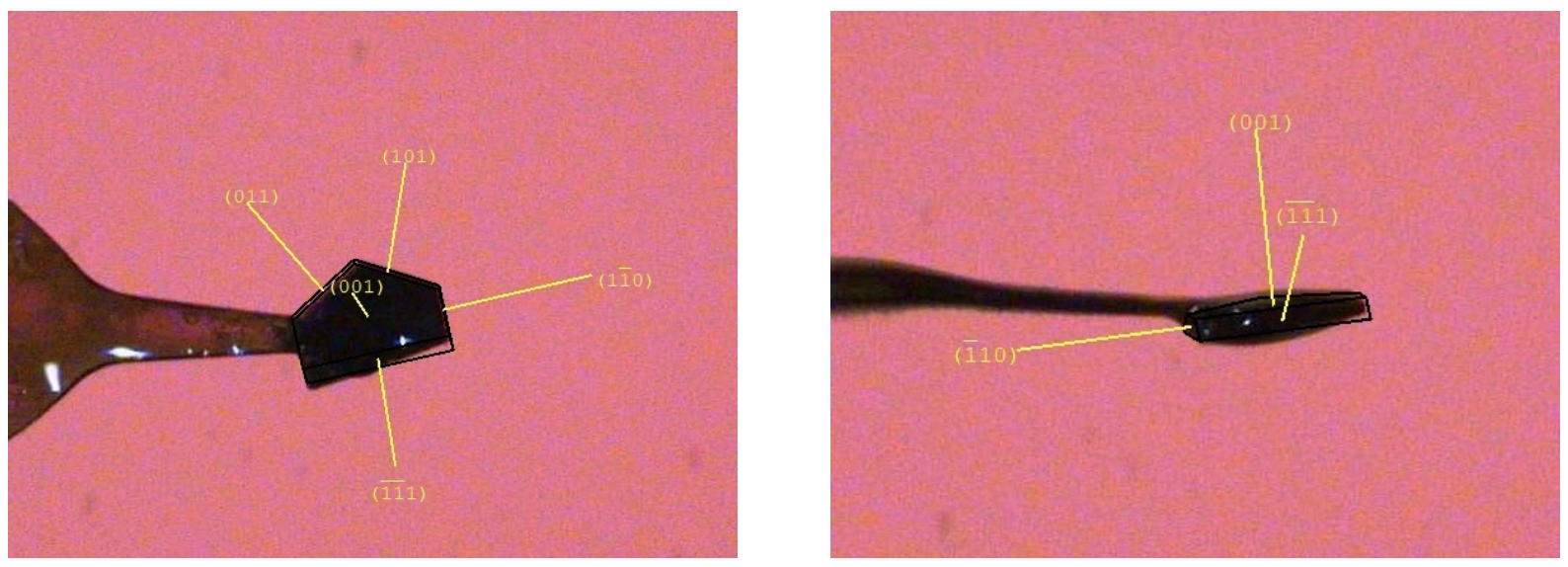

Figure S2. A single crystal of $\mathbf{2}$ after being mounted on the X-ray diffractometer, highlighting the hexagonal shape and thickness of the crystal. Left: View of the longer dimension of the crystal. Right: View of the shorter dimension of the crystal. 


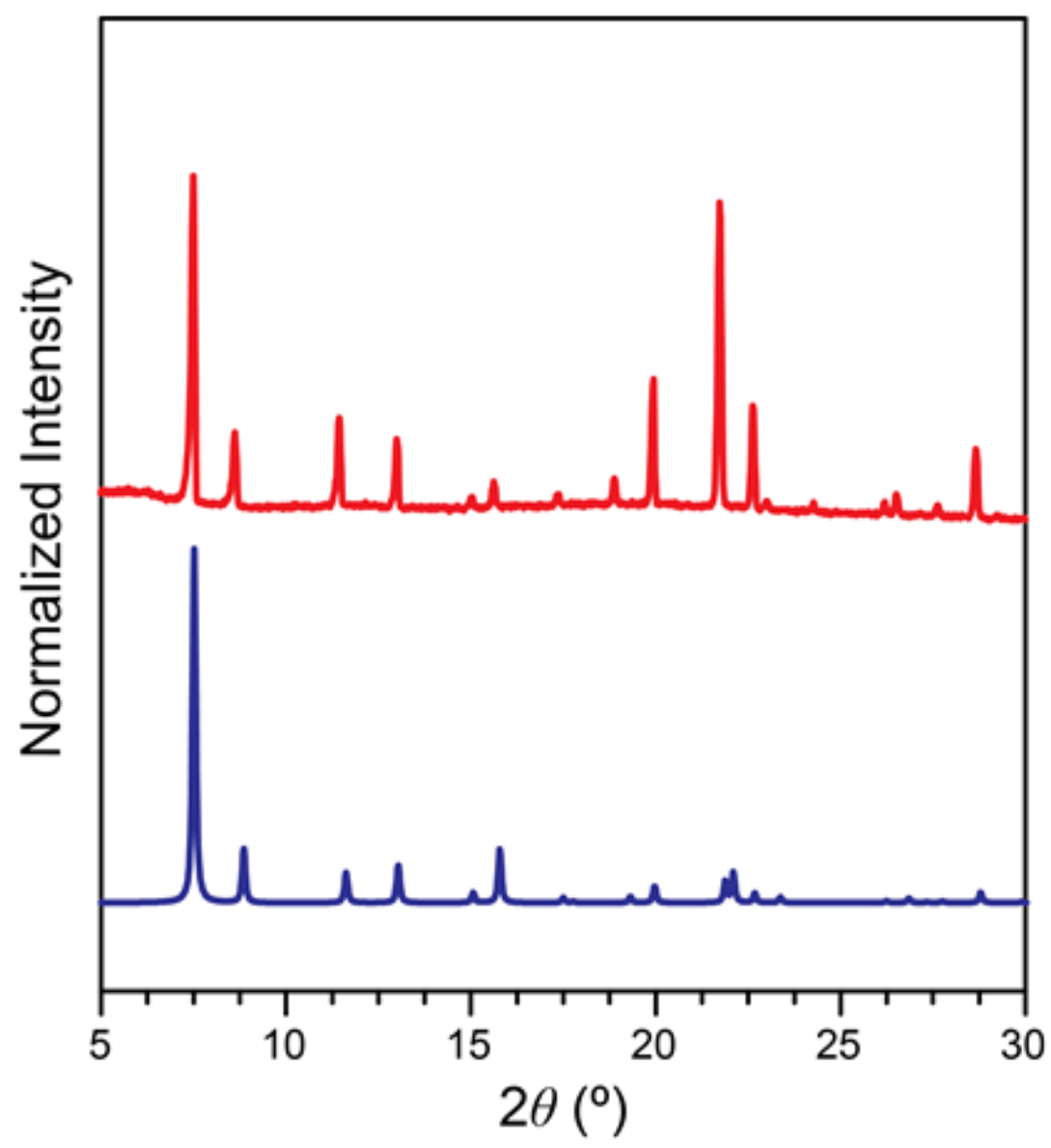

Figure S3. Powder diffraction pattern of 2 (red) collected at a wavelength of $1.54056 \AA$, and a simulated pattern of $\mathbf{2}$ obtained from single-crystal X-ray diffraction data (blue). 


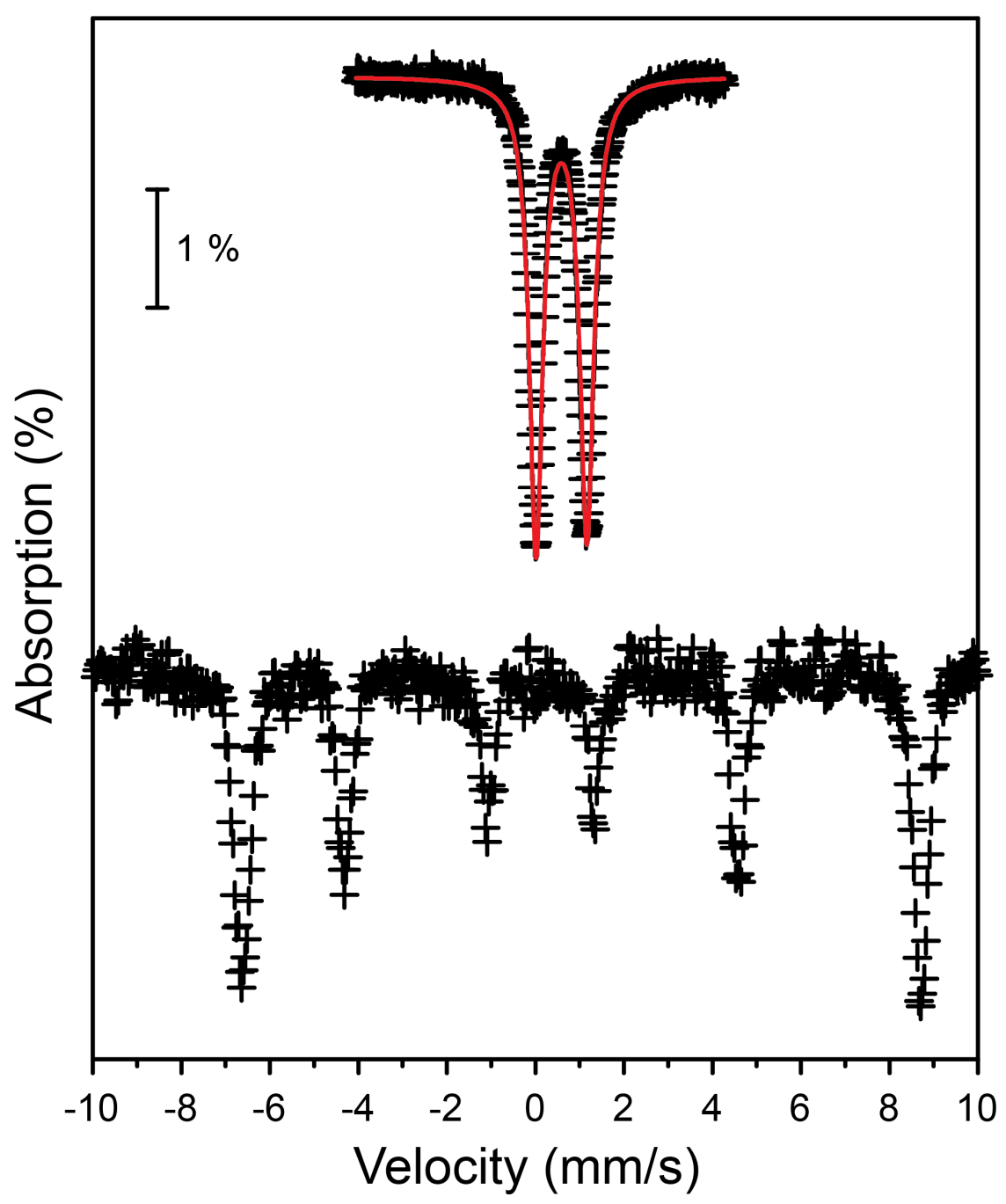

Figure S4. Mössbauer spectra collected for a crystalline sample of 2 at $80 \mathrm{~K}$ (upper) and $4 \mathrm{~K}$ (lower). Black crosses represent experimental data and the red solid line corresponds to a fit to the data, giving $\delta$ $=0.574(1) \mathrm{mm} / \mathrm{s}$ and a quadruple splitting of $\Delta E_{\mathrm{Q}}=1.125(5) \mathrm{mm} / \mathrm{s}$. 


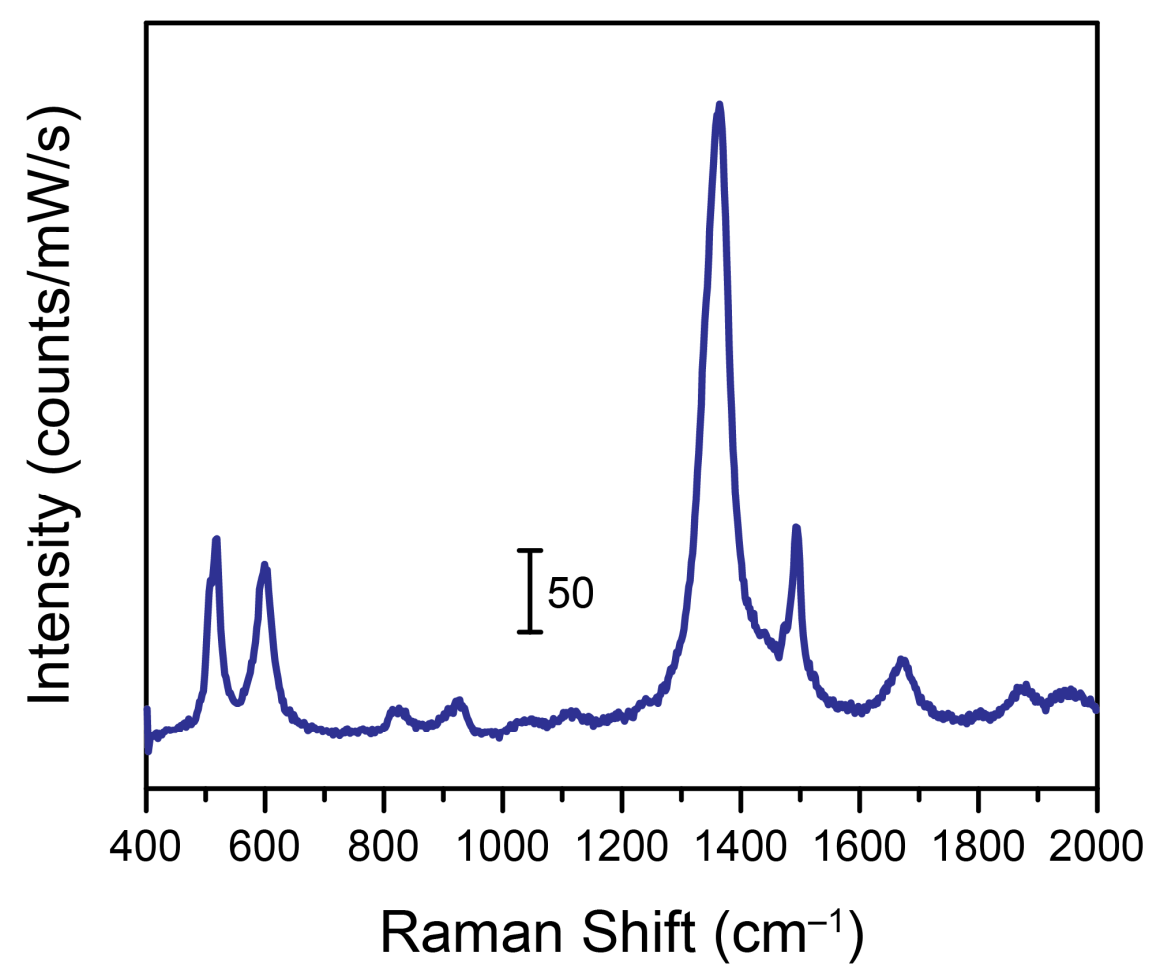

Figure S5. Raman spectrum collected for a solid sample of 2 using $473 \mathrm{~nm}$ laser excitation. Prominent vibrations are centered at $1364 \mathrm{~cm}^{-1}$ and $1494 \mathrm{~cm}^{-1}$, which are assigned to $\mathrm{C}-\mathrm{C}$ and $\mathrm{C}-\mathrm{O}$ stretching vibrations from the ligands. The Raman bands for these two vibrations are similar to those in $\mathbf{1}$, which features mixed-valent ligands with Raman bands at $1364 \mathrm{~cm}^{-1}$ and $1492 \mathrm{~cm}^{-1}$. These vibrations fall in between the vibrations for frameworks containing only $\mathrm{L}^{2-}\left(1360 \mathrm{~cm}^{-1}\right.$ and $\left.1617 \mathrm{~cm}^{-1}\right)$ and frameworks containing only $\mathrm{L}^{3-\bullet}\left(1390 \mathrm{~cm}^{-1}\right.$ and $\left.1487 \mathrm{~cm}^{-1}\right){ }^{7}$ 

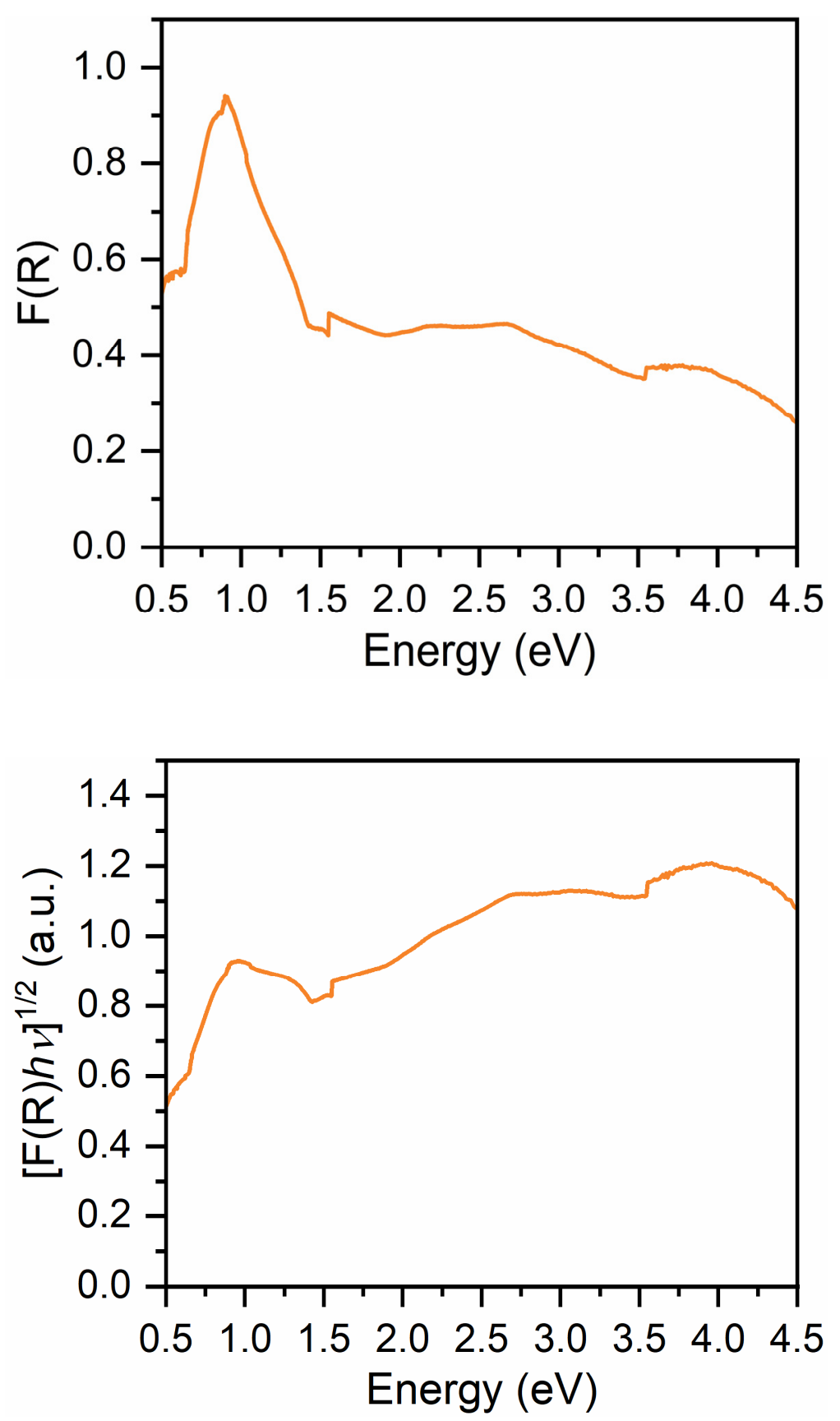

Figure S6. (Top) UV-vis-NIR diffuse reflectance spectrum for 2 collected in a $\mathrm{BaSO}_{4}$ matrix. The strong absorbance below $1 \mathrm{eV}$ corresponds to an intervalence charge transfer transition, while features above 2 $\mathrm{eV}$ correspond to charge-transfer and ligand-centered transitions. (Bottom) Tauc plot obtained from the diffuse reflectance spectrum of $\mathbf{2}$. The absorbance onset is below the limit of our detector, preventing unqualified determination of the optical band gap. An extrapolation of the nearly linear region from $0.5-$ $0.8 \mathrm{eV}$ yields an estimated band gap of $\sim 0.35 \mathrm{eV}$. 


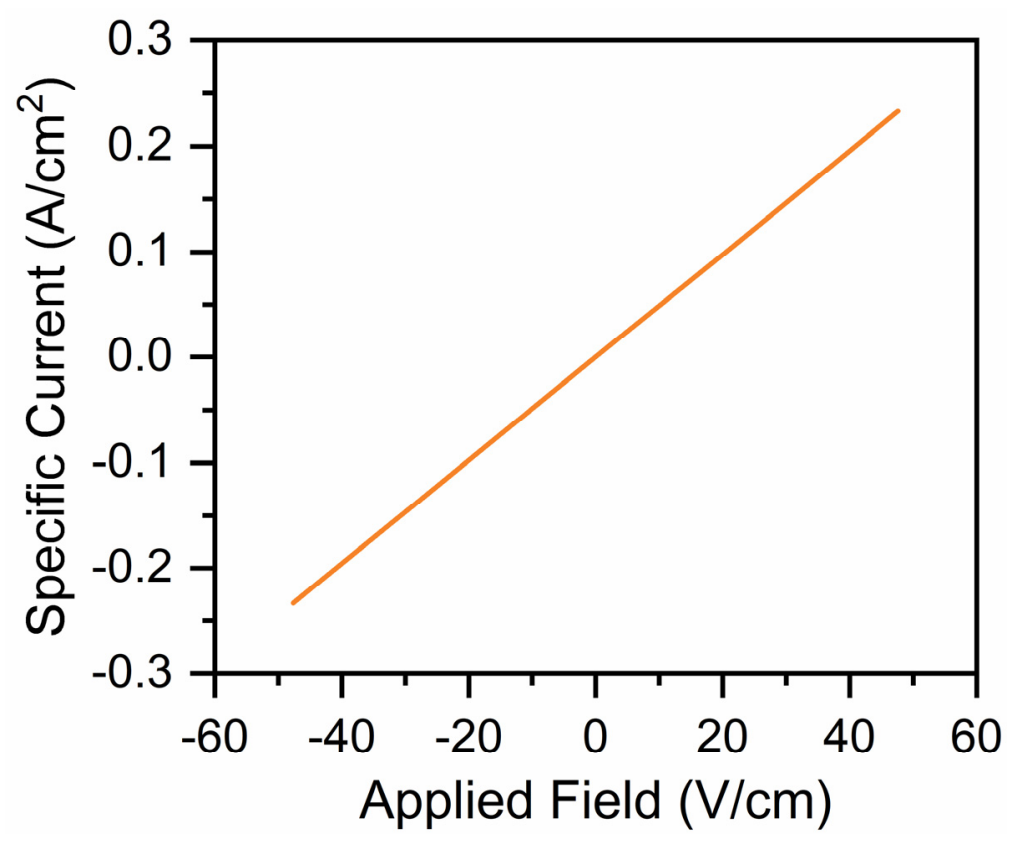

Figure S7. Pressed pellet electronic conductivity data for $\mathbf{2}$ using a previously reported screw cell setup. ${ }^{18}$ The conductivity measured here is $4.9 \times 10^{-3} \mathrm{~S} / \mathrm{cm}$, comparable to the conductivity of 1 . The cylindrical pellet had a cross-sectional area of $0.051 \mathrm{~cm}^{2}$ and a length of $0.021 \mathrm{~cm}$. 


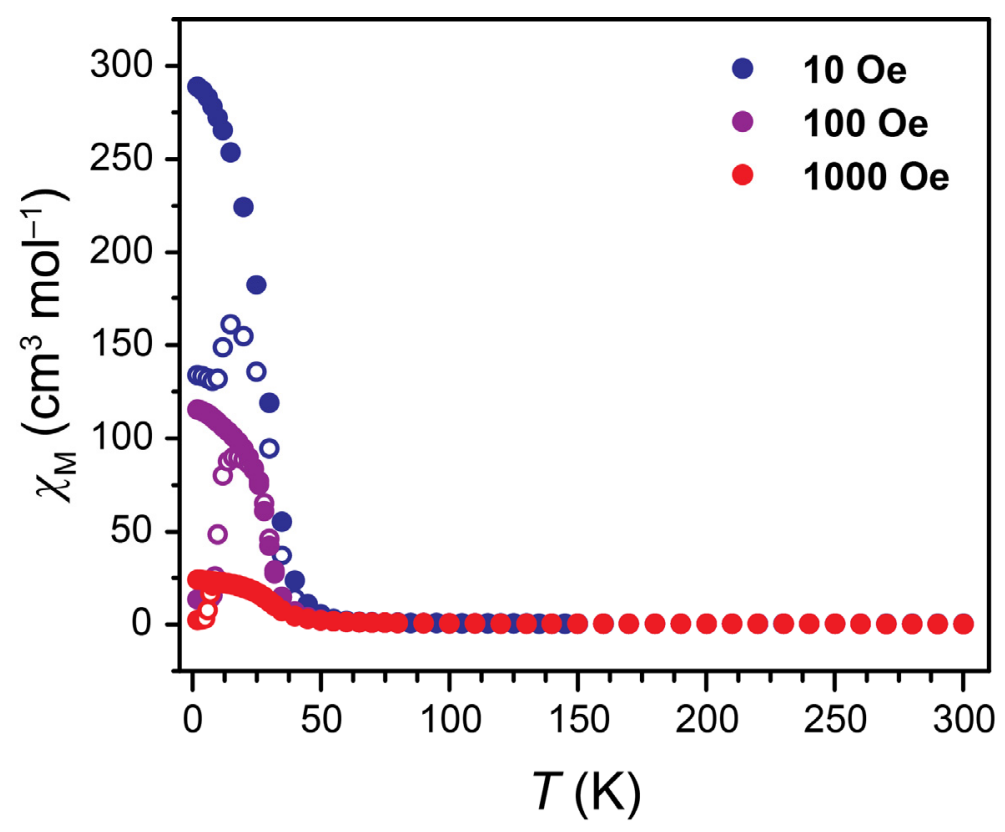

Figure S8. Variable-temperature zero-field-cooled (open circles) and field-cooled (solid circles) dc magnetic susceptibility data for an unoriented sample of $\mathbf{2}$ collected under applied magnetic fields of 10 (blue), 100 (purple), and 1000 (red) Oe.

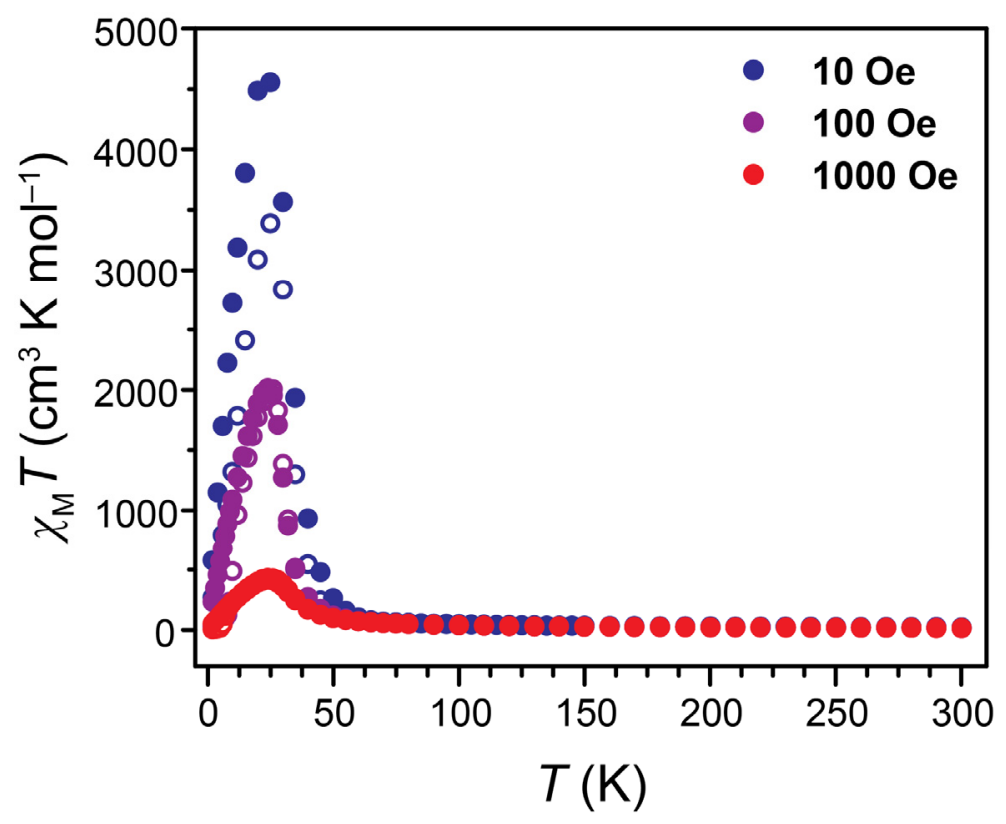

Figure S9. Plots of $\chi_{\mathrm{M}} T$ vs $T$ for an unoriented sample of $\mathbf{2}$, obtained from variable-temperature zerofield-cooled (open circles) and field-cooled (solid circles) dc magnetic susceptibility data, collected under applied magnetic fields of 10 (blue), 100 (purple), and 1000 (red) Oe. 


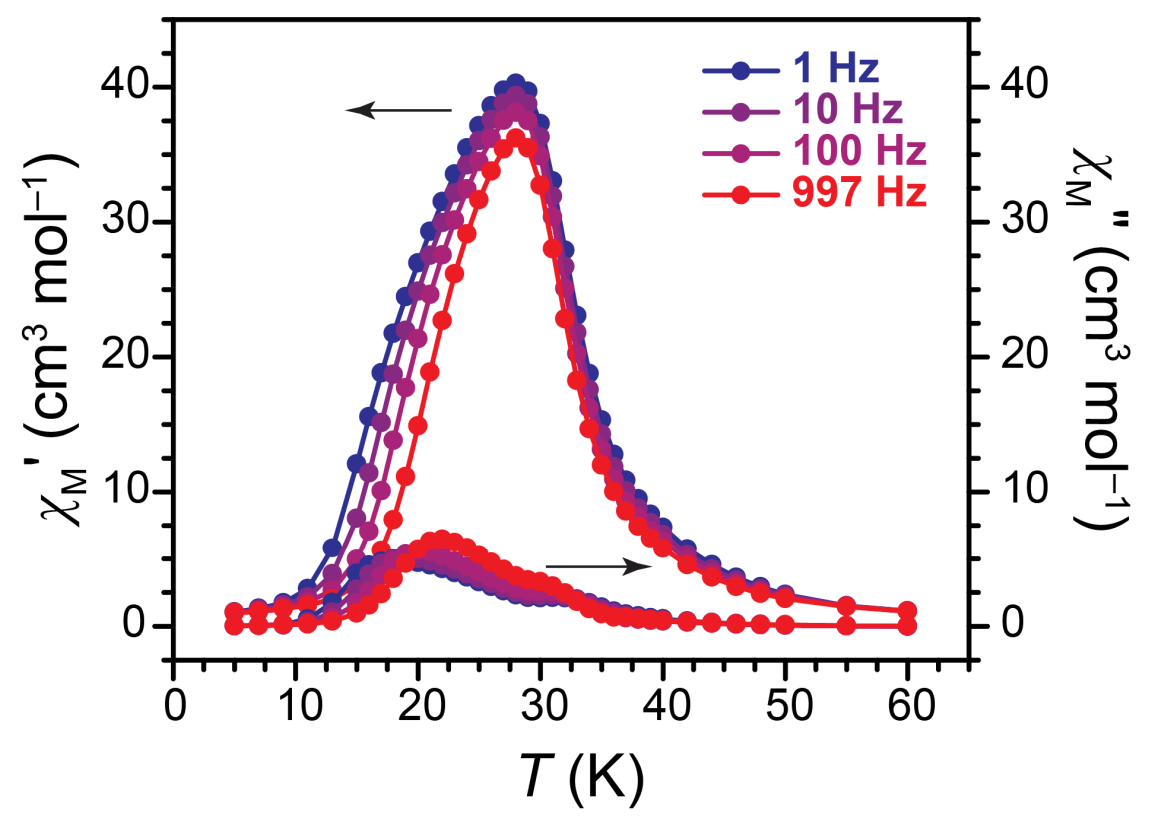

Figure S10. Variable-temperature ac magnetic susceptibility data for an unoriented sample of $\mathbf{2}$ collected under zero de magnetic field in the frequency range of 1 to $997 \mathrm{~Hz}$ (blue to red). Colored circles denote experimental data and solid lines are guides for the eye.

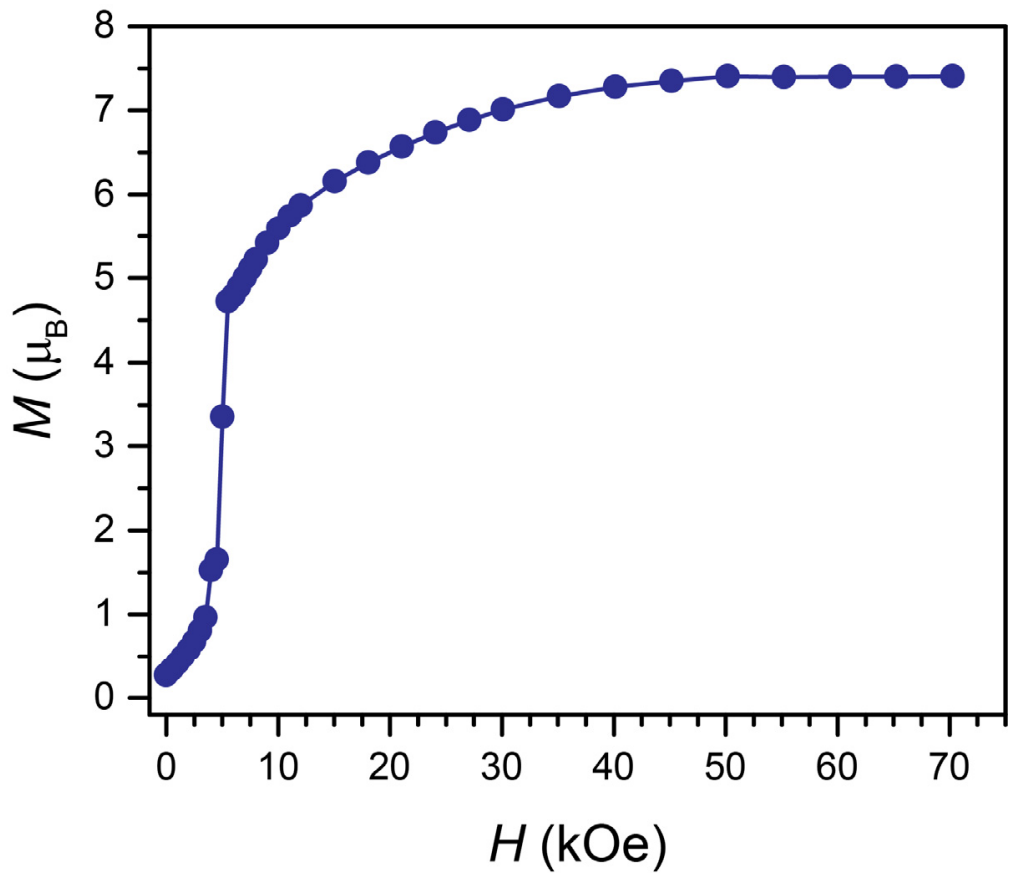

Figure S11. Variable-field dc magnetization data for an unoriented sample of an unoriented sample of 2 collected at $1.8 \mathrm{~K}$. Circles denote experimental data and solid lines are guides for the eye. 

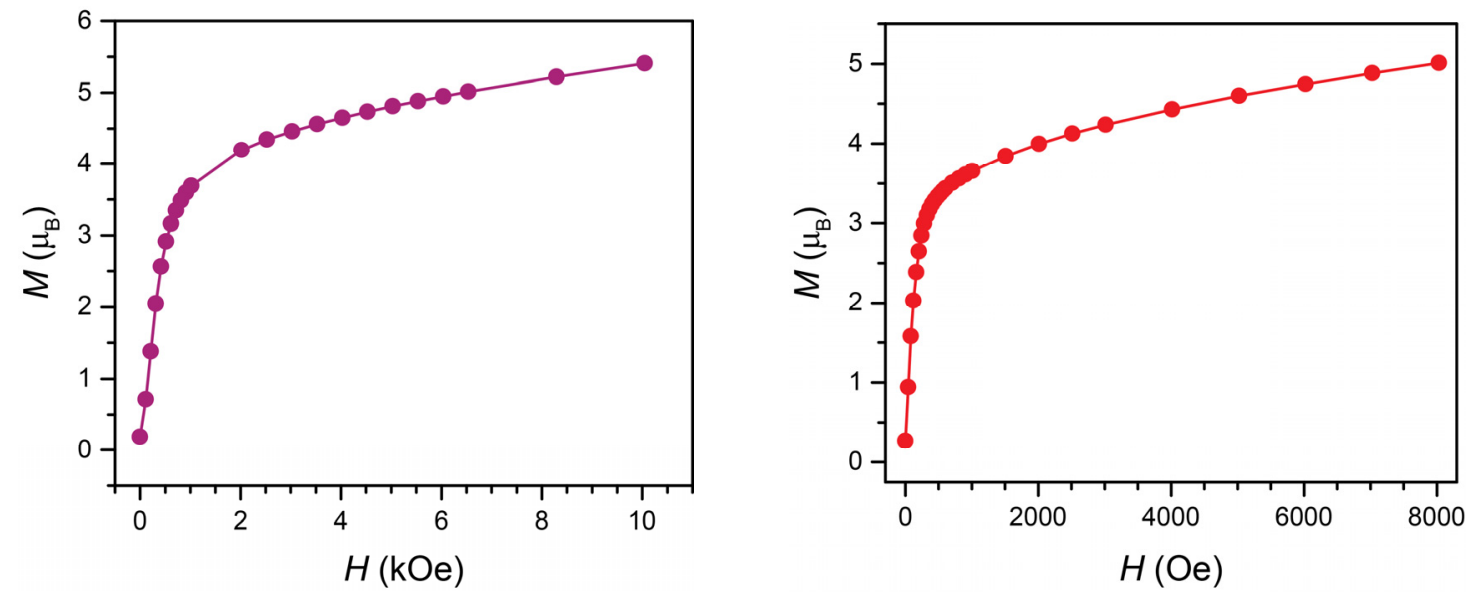

Figure S12. Variable-field dc magnetization data for an unoriented sample of 2 collected at 10 (magenta) and $20 \mathrm{~K}$ (red). Colored circles denote experimental data and solid lines are guides for the eye.
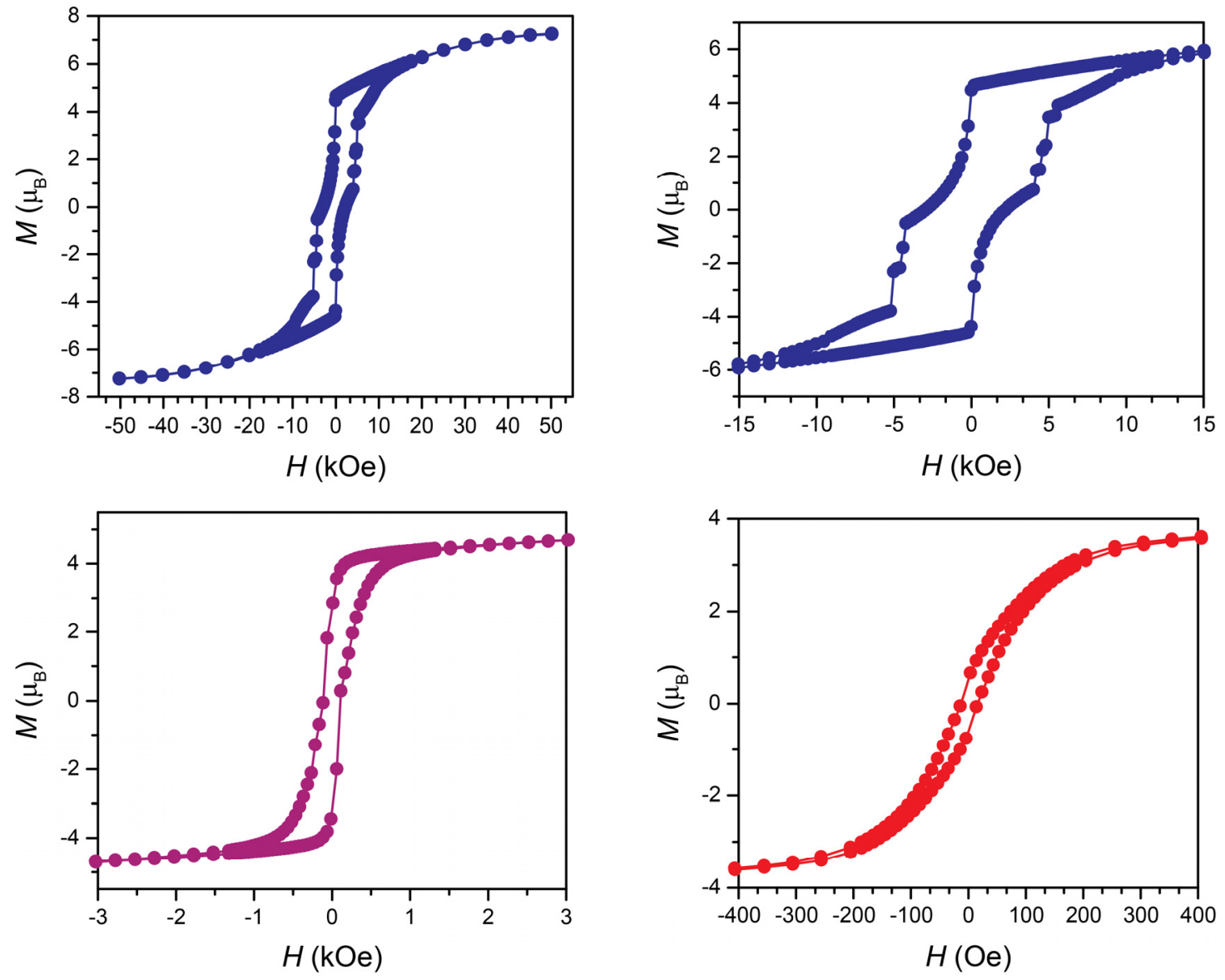

Figure S13. Variable-field magnetic hysteresis data for an unoriented sample of 2 collected at 1.8 (blue), 10 (magenta), and 20 (red) $\mathrm{K}$. Coercive fields with values of $H_{\mathrm{ci}}=3013,114,14 \mathrm{Oe}$ at 20, 10 and $1.8 \mathrm{~K}$, respectively, are observed with a field-sweep rate of $1.94,0.71,0.08 \mathrm{Oe} \mathrm{s}^{-1}$, respectively. Colored circles denote experimental data and solid lines are guides for the eye. 


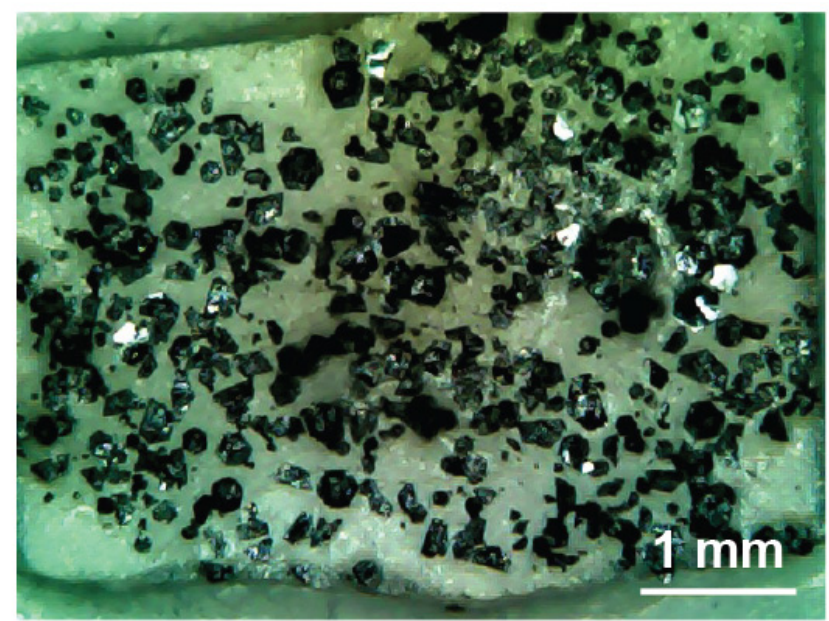

Figure S14. Photograph of an oriented single-crystal sample used for oriented-crystal magnetic measurements. Nearly all of the crystals are aligned with the $c$ axis perpendicular to the substrate (parallel to the viewing direction).

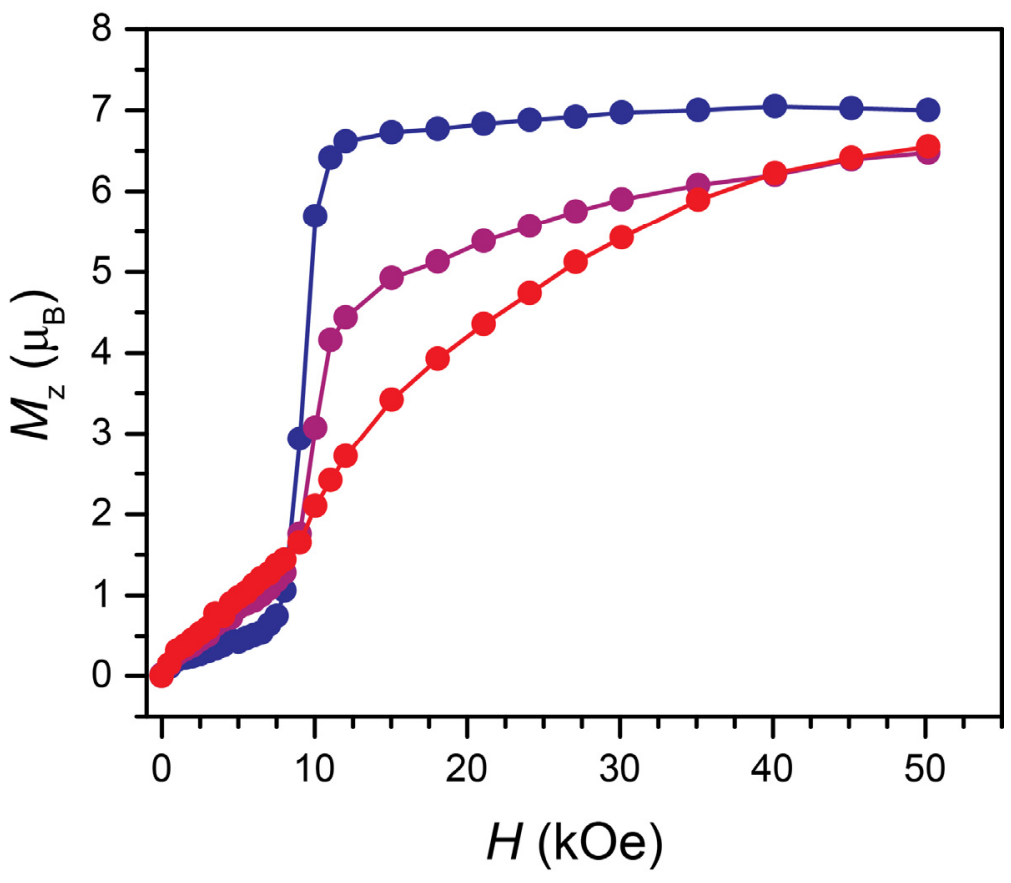

Figure S15. Variable-field dc magnetization data collected at $1.8 \mathrm{~K}$ for the oriented single-crystal sample of 2. The blue circles denote the data when the sample is in the out-of-plane geometry where the crystallographic $c$ axis is aligned with the applied magnetic field, the magenta circles denote the data when the sample is in the geometry where the crystallographic $c$ axis is aligned $45^{\circ}$ to the applied magnetic field, and the red circles denote the data when the sample is in the in-plane geometry where the crystallographic $a b$ plane is aligned with the applied magnetic field. Solid lines are guides for the eye. 


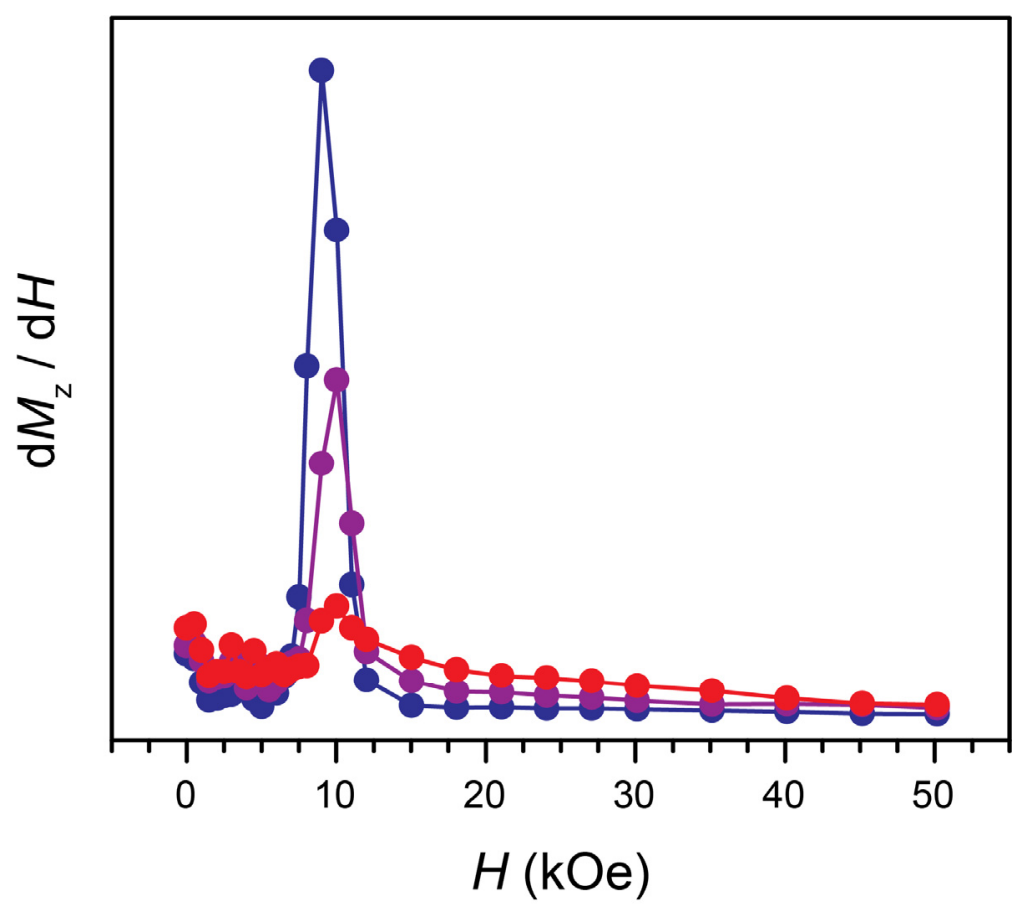

Figure S16. Plots of $\mathrm{d} M_{\mathrm{z}} / \mathrm{d} H$ vs $H$ for data collected at $1.8 \mathrm{~K}$ for the oriented single-crystal sample of 2 . The blue circles denote the data when the sample is in the out-of-plane geometry where the crystallographic $c$ axis is aligned with the applied magnetic field, the magenta circles denote the data when the sample is in the geometry where the crystallographic $c$ axis is aligned $45^{\circ}$ to the applied magnetic field, and the red circles denote the data when the sample is in the in-plane geometry where the crystallographic $a b$ plane is aligned with the applied magnetic field. Solid lines are guides for the eye. 


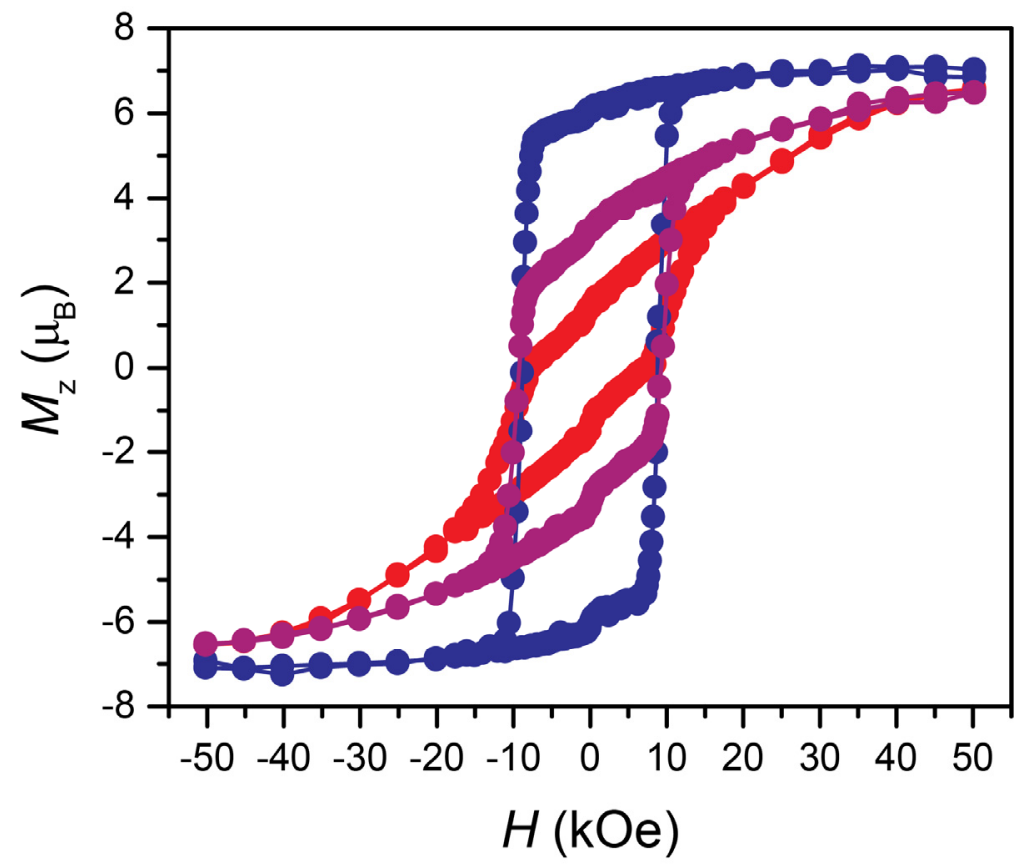

Figure S17. Variable-field magnetic hysteresis data collected at $1.8 \mathrm{~K}$ for the oriented single-crystal sample of 2 . The blue circles denote the data when the sample is in the out-of-plane geometry where the crystallographic $c$ axis is aligned with the applied magnetic field, the magenta circles denote the data when the sample is in the geometry where the crystallographic $c$ axis is aligned $45^{\circ}$ to the applied magnetic field, and the red circles denote the data when the sample is in the in-plane geometry where the crystallographic $a b$ plane is aligned with the applied magnetic field. Solid lines are guides for the eye. 


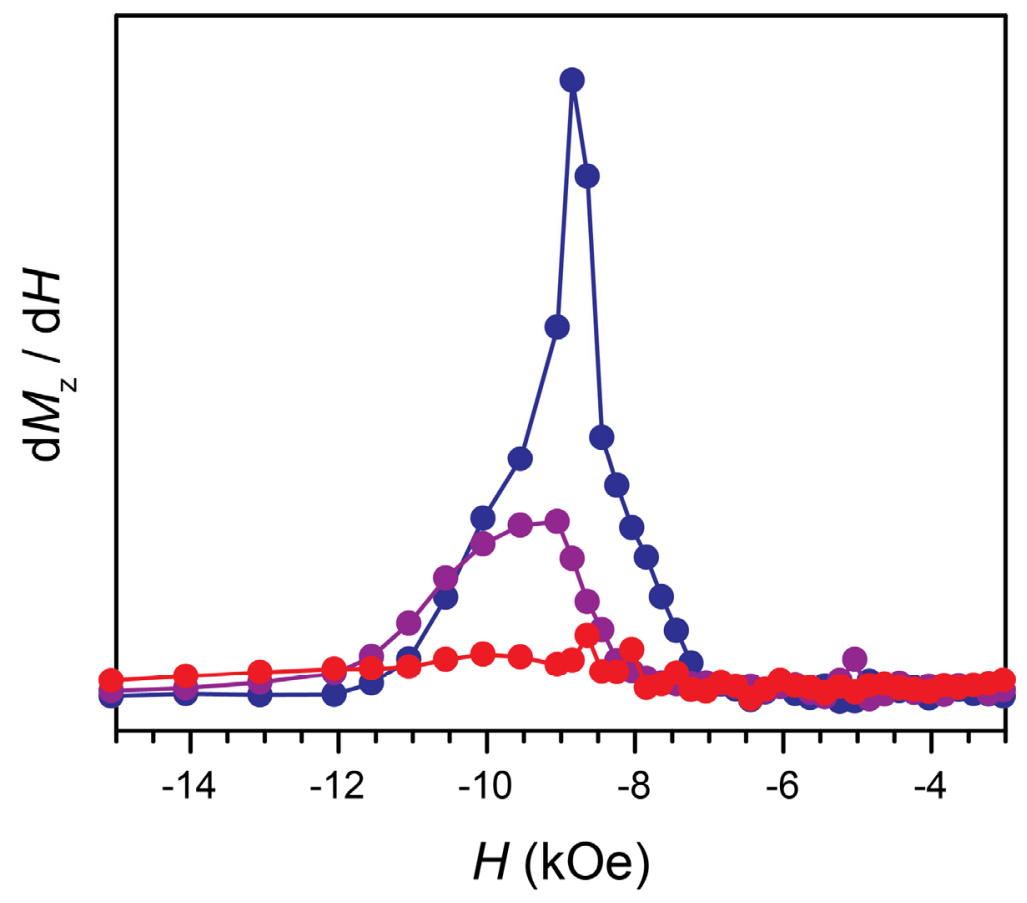

Figure S18. Switching-field distribution data collected at $1.8 \mathrm{~K}$ for the oriented single-crystal sample of 2. The blue circles denote the data when the sample is in the out-of-plane geometry where the crystallographic $c$ axis is aligned with the applied magnetic field, the magenta circles denote the data when the sample is in the geometry where the crystallographic $c$ axis is aligned $45^{\circ}$ to the applied magnetic field, and the red circles denote the data when the sample is in the in-plane geometry where the crystallographic $a b$ plane is aligned with the applied magnetic field. Solid lines are guides for the eye. 


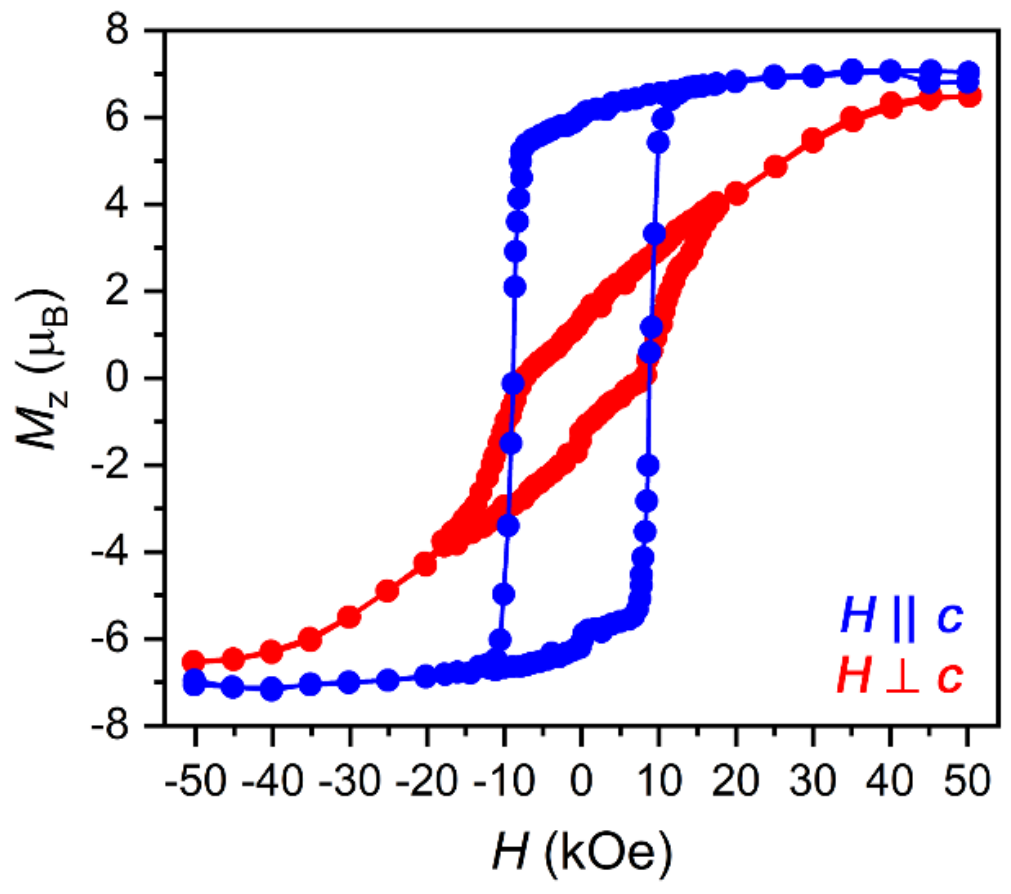

Figure S19. Variable-angle magnetic hysteresis data for the oriented single-crystal sample of $\mathbf{2}$ collected at $1.8 \mathrm{~K}$ along two different crystallographic directions. Red and blue lines are guides to the eye. 


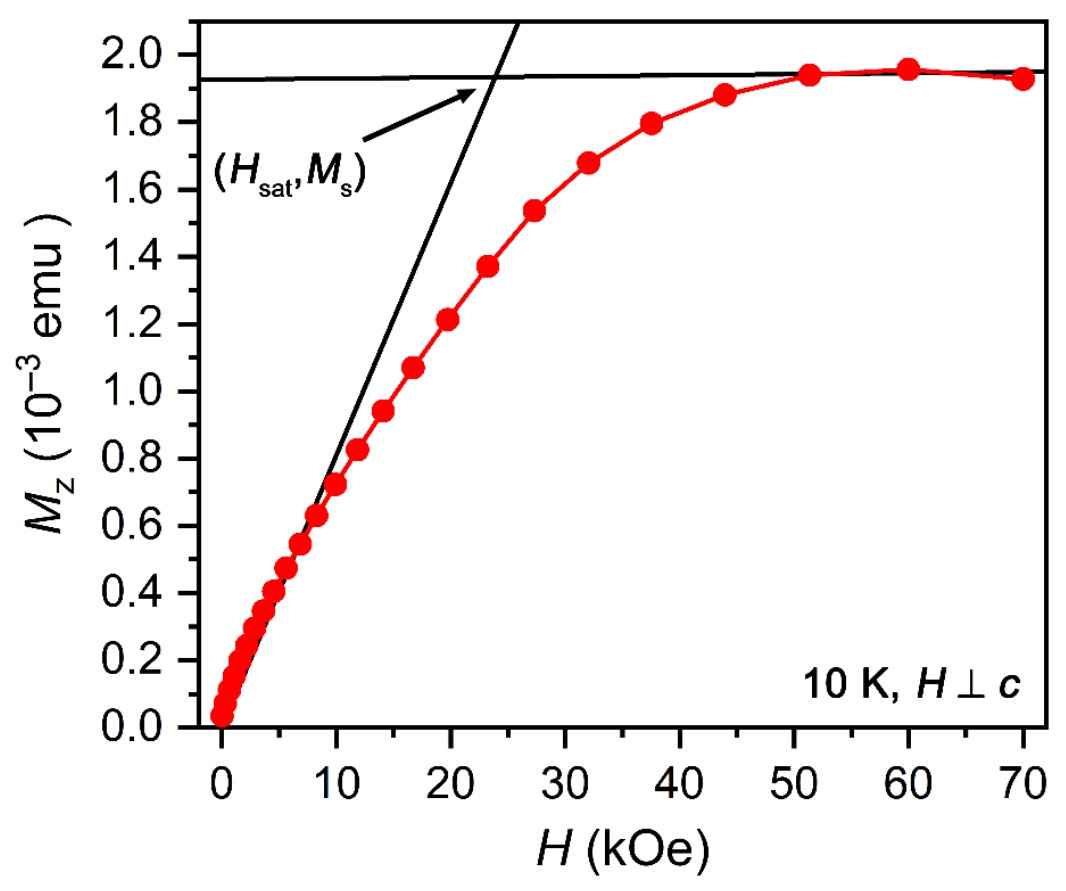

Figure S20. Plot of magnetization vs applied field for oriented crystals of 2 at $10 \mathrm{~K}$. Data was corrected to remove a sharp increase in the magnetization at low fields, corresponding to a small number of crystals with $H \| c$. Linear fits to the low- and high-field regime were used to determine $H_{\mathrm{c}}$ and $M_{\mathrm{s}}$ for determination of $K_{\mathrm{u}}$ using the Stoner-Wohlfarth method.

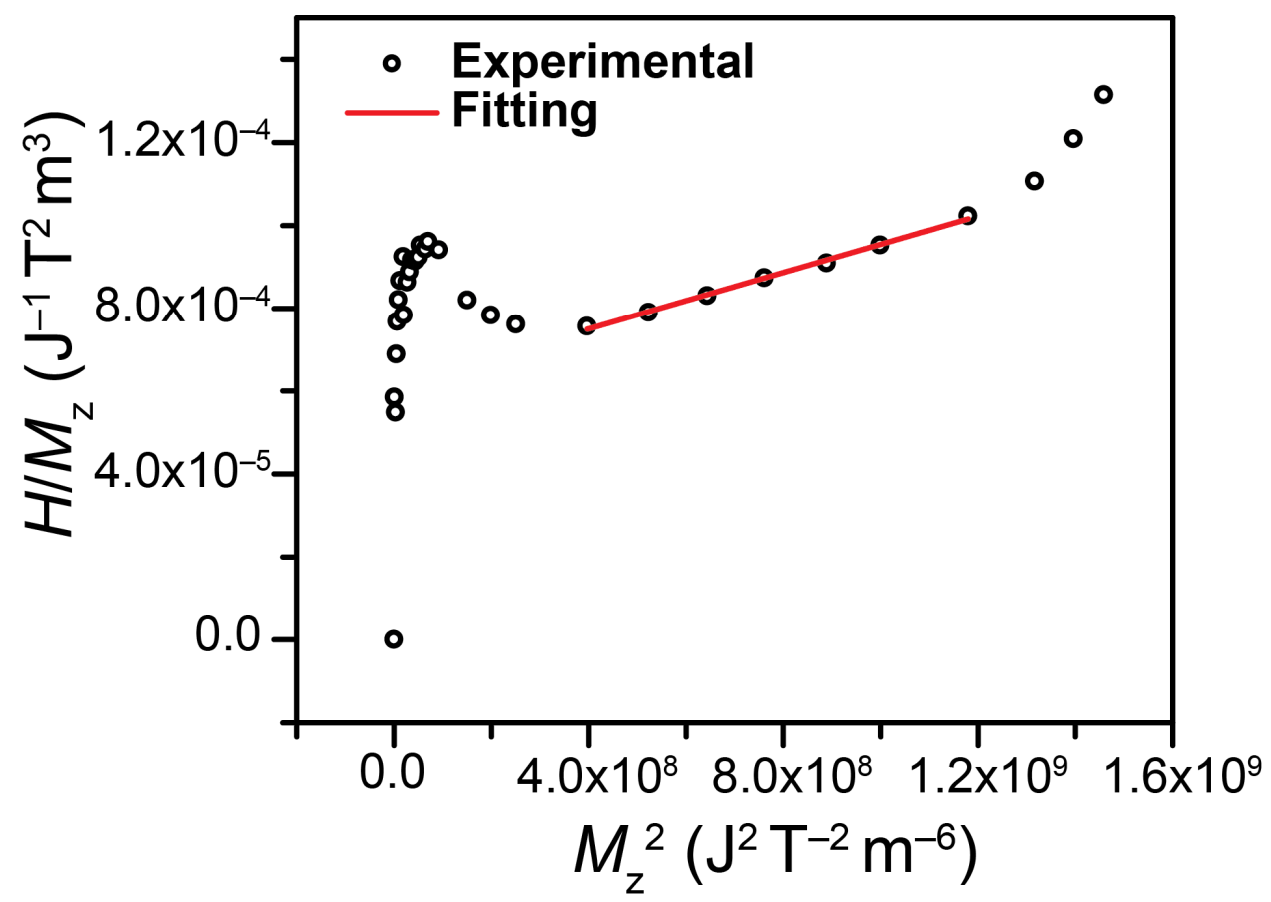

Figure S21. Plot of $H / M_{\mathrm{z}}$ vs $M_{\mathrm{z}}^{2}$ based on the Sucksmith-Thompson method. Open circles denote experimental data, red solid line corresponsd to a fit of the data to equation S2. 
a)

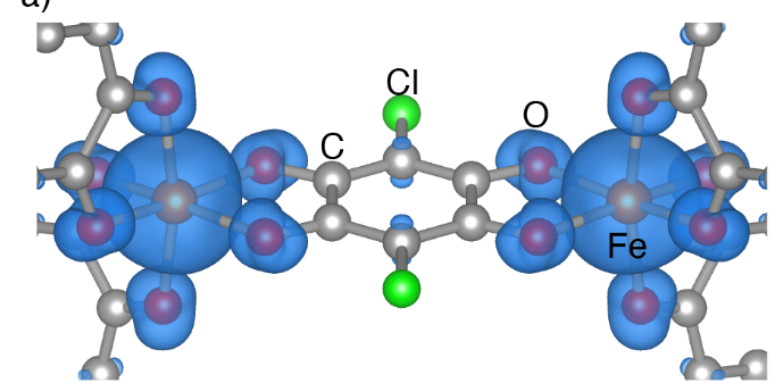

b)

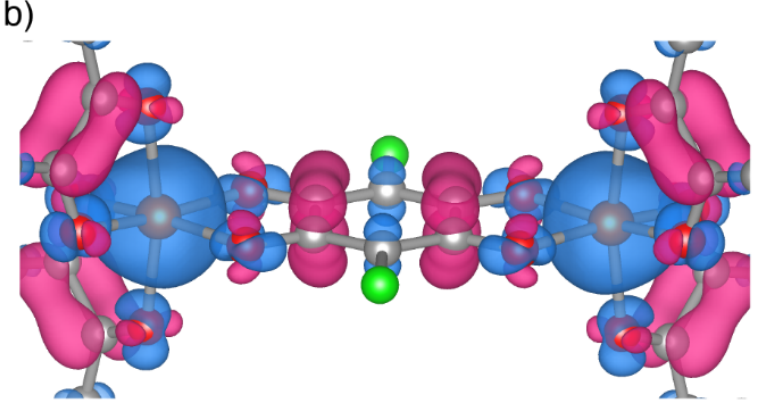

Figure S22. Spin density plots for the neutral (a) and dianionic (b) models of the unit cell of 2, neglecting spin-orbit coupling. The $\alpha$ (spin up) and $\beta$ (spin down) densities are shown in blue and purple, respectively. 
a)

b)
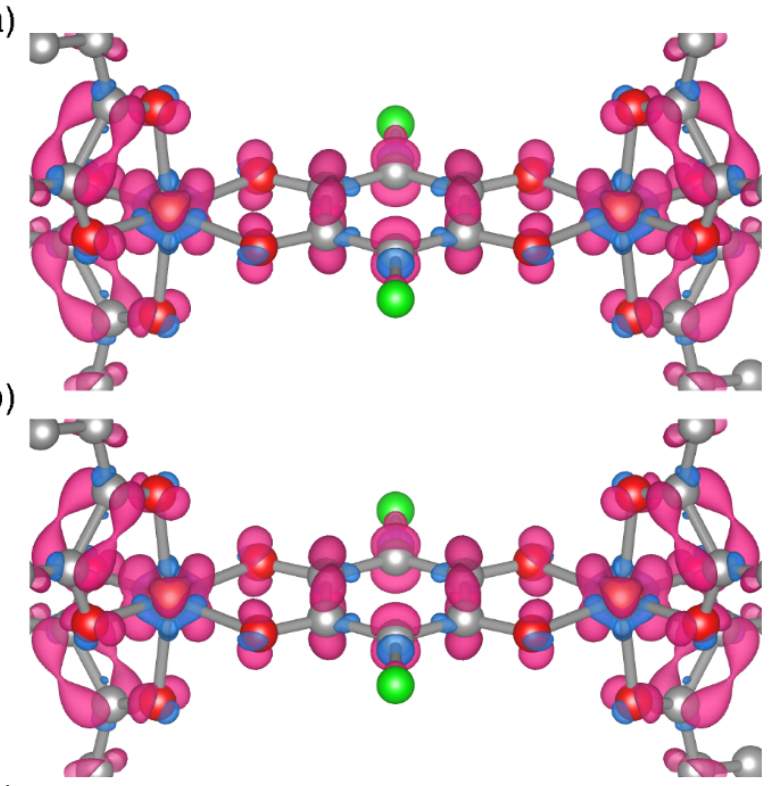

c)

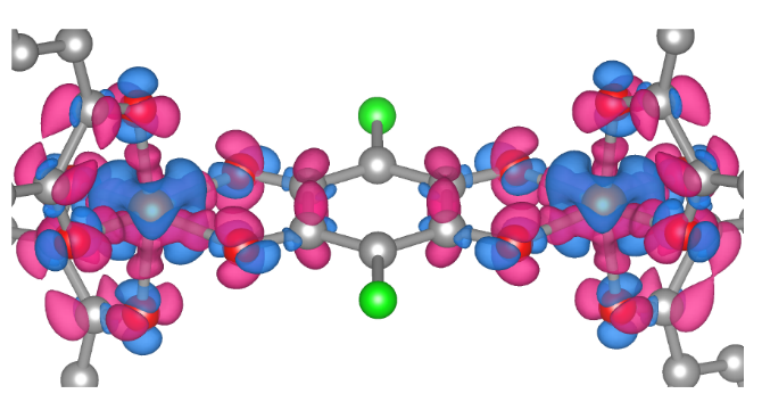

Figure S23. Charge density difference plots for the dianionic vs neutral models of the unit cell, calculated with spin-orbit coupling, and with the magnetization aligned along the crystallographic $a$-axis (a) or $c$-axis (b). Pink and blue lobes correspond to regions of increased and decreased charge density, respectively, in the dianionic model relative to the neutral model. (c) Charge density difference plot between the dianionic models with the magnetization aligned along the crystallographic $a$ - or $c$-axis. Pink and blue lobes correspond to regions of increased and decreased charge density, respectively, in the dianionic model relative to the neutral model. The isolevels are $0.003 \mathrm{e} / \AA^{3}$ for a and $\mathrm{b}$, and $3.5 \times 10^{-5} \mathrm{e} / \AA^{3}$ for $\mathrm{c}$. 
a) without SOC

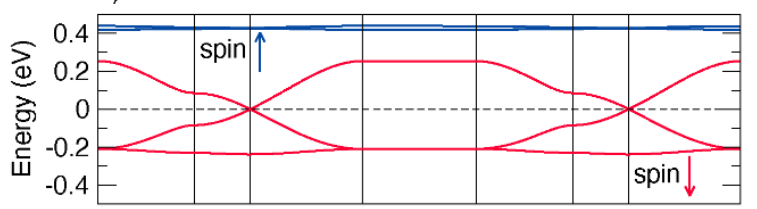

b) SOC $m$ parallel to a

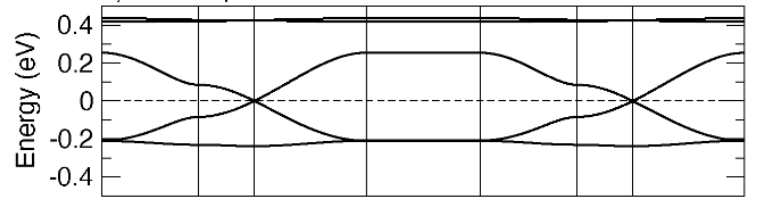

c) SOC $m$ parallel to $c$

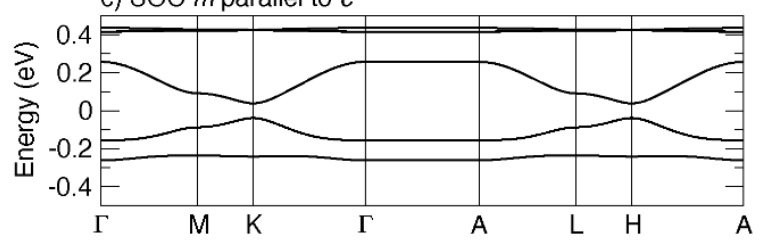

d) wannierization

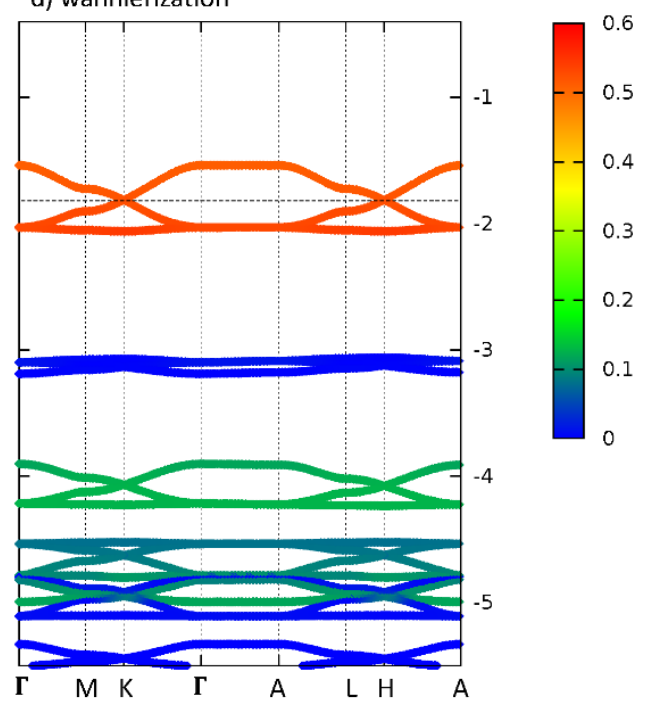

Figure S24. Ferromagnetic band structure of 2 neglecting spin-orbit coupling (a) or including spin-orbit coupling with the magnetization aligned with the crystallographic $a$-axis (b) or $c$-axis (c). (d) The Wannierinterpolation of the spin-down channel in the band structure of 2. The color field is a projection of the Wannier function shown in the main text (Figure 5) onto the Bloch states. The dashed line indicates the Fermi level.

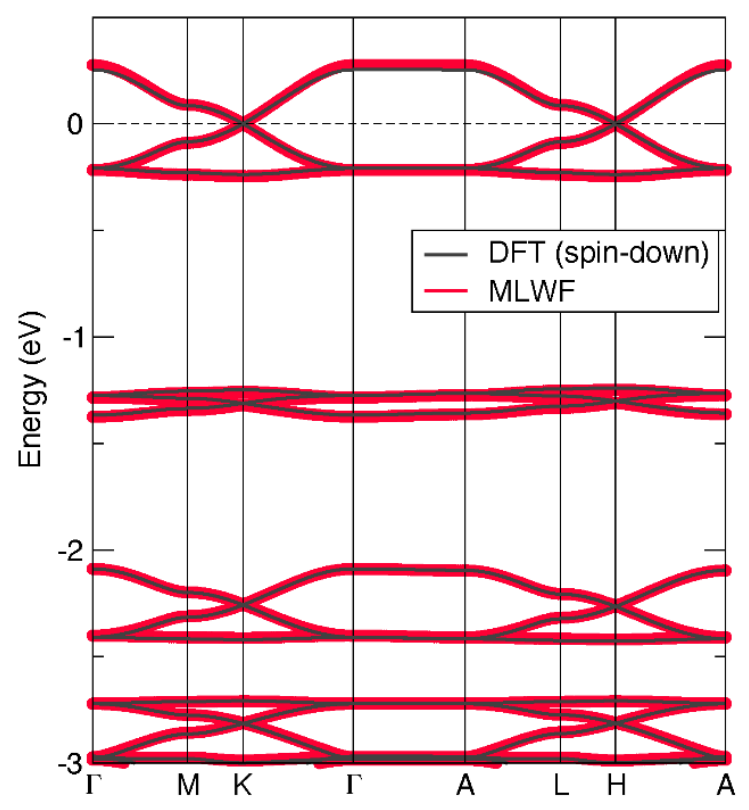

Figure S25. Comparison between the spin-down bands of the band structure computed with DFT and the MLWF bands, with spin-orbit coupling neglected in both cases. Excellent agreement is observed between the two models. The dashed line indicates the Fermi level. 


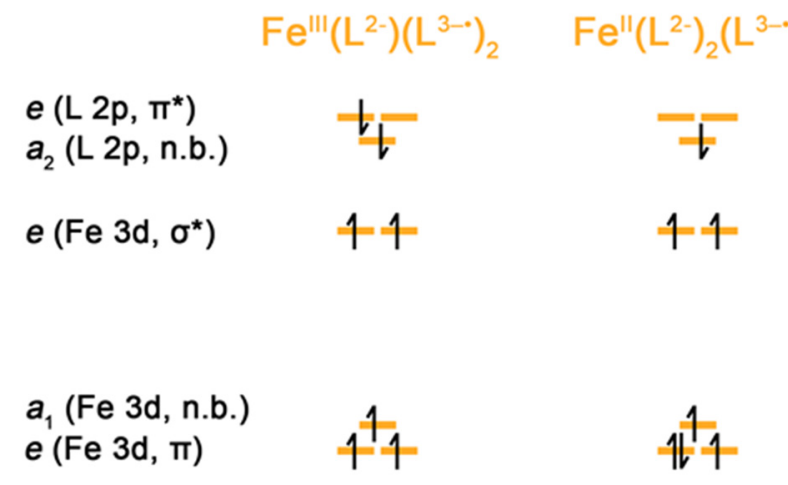

Figure S26. Molecular orbital levels for a single Fe center within 2, using a local $D_{3}$ symmetry. The orbital filling on the left most closely matches the electronic structure of $\mathbf{2}$, with high spin $\mathrm{Fe}^{\mathrm{III}}$ ions. The orbital filling on the right illustrates the excited state generated by ligand-to-metal charge transfer, containing an $\mathrm{Fe}^{\mathrm{II}}$ center with unquenched orbital angular momentum. The presence of metal-ligand covalency in 2 introduces $\beta$-spin density on the Fe centers, analogous to the excited state observed here, explaining the magnetocrystalline anisotropy observed experimentally and computationally. The labels on the left indicate the symmetry, primary orbital contributor, and bonding interaction for each orbital level. 


\section{References}

(1) APEX2, version 2009.1; Bruker Analytical X-ray Systems, Inc.: Madison, WI, 2009.

(2) Sheldrick, G. M. SADABS, version 2.03; Bruker Analytical X-ray Systems, Inc.: Madison, WI, 2000.

(3) Sheldrick, G. M. SHELXTL, version 6.12; Bruker Analytical X-ray Systems, Inc.: Madison, WI, 2000.

(4) Dolomanov, O. V.; Bourhis, L. J.; Gildea, R. J.; Howard, J. A. K.; Puschmann, H. OLEX2: A Complete Structure Solution, Refinement and Analysis Program. J. Appl. Crystallogr. 2009, 42, 339341.

(5) Bain, G. A.; Berry, J. F. Diamagnetic Corrections and Pascal's Constants. J. Chem. Educ. 2008, 85, 532-536.

(6) Perdew, J. P.; Ernzerhof, M.; Burke, K. Rationale for Mixing Exact Exchange with Density Functional Approximations. J. Chem. Phys. 1996, 105, 9982-9985.

(7) Kresse, G.; Furthmuller, J. Efficiency of Ab-Initio Total Energy Calculations for Metals and Semiconductors Using a Plane-Wave Basis Set. Comput. Mater. Sci. 1996, 6, 15-50.

(8) Blöchl, P. E.; Jepsen, O.; Andersen, O. K. Improved Tetrahedron Method for Brillouin-Zone Integrations. Phys. Rev. B 1994, 49, 16223.

(9) Grimme, S.; Antony, J.; Ehrlich, S.; Krieg, S. A Consistent and Accurate Ab Initio Parametrization of Density Functional Dispersion Correction (DFT-D) for the 94 Elements H-Pu. J. Chem. Phys. 2010, $132,154104$.

(10) Franchini, C.; Kováčik, R.; Marsman, M.; Sathyanarayana Murthy, S.; He, J.; Ederer, C.; Kresse, G. Maximally Localized Wannier Functions in $\mathrm{LaMnO}_{3}$ within $\mathrm{PBE}+U$ Hybrid Functionals and Partially Self-Consistent GW: An Efficient Route to Construct $A b$ Initio Tight-Binding Parameters for $e_{g}$ Perovskites. J. Phys. Condens. Matter 2012, 24, 235602.

(11) Mostofi, A. A.; Yates, J. R.; Pizzi, G.; Lee, Y.-S.; Souza, I.; Vanderbilt, D.; Marazari, N. An Updated Version of wannier90: A Tool for Obtaining Maximally-Localised Wannier Functions. Comp. Phys. Commun. 2014, 185, 2309-2310.

(12) Mydosh, J. A. Spin Glasses: An Experimental Introduction; Taylor \& Francis: London, 1993.

(13) (a) Shilov, G. V.; Nikitina, Z. K.; Ovanesyan, N. S.; Aldoshin, S. M.; Makhaev, V. D. Phenazineoxonium Chloranilateomanganate and Chloranilatoferrate: Synthesis, Structure, Magnetic Properties, and Mössbauer Spectra. Russ. Chem. Bull. 2011, 60, 1290-1219. (b) Jeon, I.-R.; Negru, B.; Van Duyne, R. P.; Harris, T. D. A 2D Semiquinone Radical-Containing Microporous Magnet with Solvent-Induced Switching from $T_{\mathrm{c}}=26$ to $80 \mathrm{~K}$. J. Am. Chem. Soc. 2015, 137, 15699-15702. (c) DeGayner, J. A.; Jeon, I.-R.; Sun, L.; Dincă, M.; Harris, T. D. 2D Conductive Iron-Quinoid Magnets Ordering up to $T_{\mathrm{c}}=105 \mathrm{~K}$ via Heterogenous Redox Chemistry. J. Am. Chem. Soc. 2017, 139, 41754184. (d) Kingsbury, C. J.; Abrahams, B. F.; D’Alessandro, D. M.; Hudson, T. A.; Murase, R.; Robson, R.; White, K. F. Role of $\mathrm{Net}_{4}{ }^{+}$in Orienting and Locking Together $\left[\mathrm{M}_{2} \mathrm{lig}_{3}\right]^{2-}(6,3)$ Sheets $\left(\mathrm{H}_{2} \mathrm{lig}=\right.$ Chloranilic or Fluoranilic Acid) to Generate Spacious Channels Perpendicular to the Sheets. Cryst. Growth Des. 2017, 17, 1465-1470. (e) Benmansour, S.; Abhervé, A.; Gómez-Claramunt, P.; VallésGarcía, C.; Gómez-García, C. J. Nanosheets of Two-Dimensional Magnetic and Conducting Fe(II)/Fe(III) Mixed-Valence Metal-Organic Frameworks. ACS. Appl. Mater. Interfaces 2017, 9, 26210-26218. (f) Chen, J.; Sekine, Y.; Komatsumaru, Y.; Hayami, S.; Miyasaka, H. Thermally Induced Valence Tautomeric Transition in a Two-Dimensional Fe-Tetraoxolene Honeycomb Network. Angew. Chem. Int. Ed. 2018, 57, 12043-12047. (g) Sahadevan, S. A.; Abhervé, A.; Monni, N.; Sáenz de Pipaón, C.; Galán-Mascaros, J. R.; Warenborgh, J. C.; Vieira, B. J. C.; Auban-Senzier, P.; Pillet, S.; Bendeif, E.-E.; Alemany, P.; Canadell, E.; Mercuri, M. L.; Avarvari, N. Conducting Anilate-Based Mixed-Valence Fe(II)Fe(III) Coordination Polymer: Small-Polaron Hopping Model for Oxalate-Type Fe(II)Fe(III) 2D Networks. J. Am. Chem. Soc. 2018, 140, 12611. (h) Chen, J.; 
Taniguchi, K.; Sekine, Y.; Miyasaka, H. Electrochemical Development of Magnetic Long-Range Correlations with $T_{\mathrm{C}}=128 \mathrm{~K}$ in a Tetraoxolene-Bridged Fe-Based Framework. Jour. Magn. Magn. Mater. 2018, 494, 165818. (i) van Koeverden, M. P. Abrahams, B. F.; D’Alessandro, D. M.; Doheny, P. W.; Hua, C.; Hudson, T. A. Jameson, G. N. L.; Murray, K. S.; Phonsri, W.; Robson, R.; Sutton, A. L. Tuning Charge-State Localization in a Semiconductive Iron(III)-Chloranilate Framework Magnet Using a Redox-Active Cation. Chem. Mater. 2020, 32, 7551-7563. (j) Chen, J.; Tanaguchi, K.; Sekine, Y.; Miyasaka, H. Magnetic Phase Switching Performance in an Fe=Tetraoxolene-Layered MetalOrganic Framework via Electrochemical Cycling. Inorg. Chem. 2021, 60, 9456-9460.

(14) (a) Morin, B. G. Zhou, P.; Hahm, C.; Epstein, A. J.; Miller, J. S. Complex ac Susceptibility Studies of the Disordered Molecular Based Magnets V(TCNE)x: Role of Spinless Solvent. J. Appl. Phys. 1993, 73, 5648-5650. (b) Tsurkan, V.; Hemberger, J.; Klemm, M.; Klimm, S.; Loidl, A.; Horn, S.; Tidecks, R. Ac susceptibility of Ferrimagnetic $\mathrm{FeCr}_{2} \mathrm{~S}_{4}$ Single Crystals. J. Appl. Phys. 2001, 90, 4639-4644. (c) Clérac, R.; O’Kane, S.; Cowen, J.; Ouyang, X.; Heintz, R.; Zhao, H.; Bazile, M. J.; Dunbar, K. R. Glassy Magnets Composed of Metals Coordinated to 7,7,8,8-tetracyanoquinodimethane: M(TCNQ)2 (M = Mn, Fe, Co, Ni). Chem. Mater. 2003, 2, 1840-1850. (d) Sarkar, T.; Pralong, V.; Caignaert, V.; Raveau, B. Competition Between Ferrimagnetism and Magnetic Frustration in Zinc-Substituted $\mathrm{YBaFe}_{4} \mathrm{O}_{7}$. Chem. Mater. 2010, 22, 2885-2891. (e) Pedersen, K. S.; Perlepe, P.; Aubrey, M. L.; Woodruff, D. N.; Reyes-Lillo, S. E.; Reinholdt, A.; Voigt, L.; Li, Z.; Borup, K.; Rouzières, M.; Samohvalov, D.; Wilhelm, F.; Rogalev, A.; Neaton, J. B.; Long, J. R.; Clérac, R. Formation of the Layered Conductive Magnet $\mathrm{CrCl}_{2}$ (pyrazine) 2 Through Redox-Active Coordination Chemistry. Nat. Chem. 2020, 10, 1056-1061.

(15) Carrasco, J. A.; Cardona-Serra, S.; Modesto Clemente-Juan, J.; Gaita-Arino, A.; Abellán, G.; Coronado, E. Deciphering the Role of Dipolar Interactions in Magnetic Layered Double Hydroxides. Inorg. Chem. 2018, 57, 2013-2022.

(16) Lee, K.; Dismukes, A. H.; Telford, E. J.; Wiscons, R. A.; Wang, J.; Xu, X.; Nuckolls, C.; Dean, C. R.; Roy, X.; Zhu, X. Magnetic Order and Symmetry in the 2D Semiconductor CrSBr. Nano Lett. 2021, 8, 3511-3517.

(17) (a) Sucksmith, W.; Thompson, J. E. The Magnetic Anisotropy of Cobalt. Proc. R. Soc. London, Ser. A 1954, 225, 362-375. (b) Bolyachkin, A. S.; Neznakhin, D. S.; Bartashevich, M. I. The Effect of Magnetization Anisotropy and Paramagnetic Susceptibility on the Magnetization Process. J. Appl. Phys. 2015, 118, 213902.

(18) Darago, L. E.; Aubrey, M. A.; Yu, C. J.; Gonzalez, M. I.; Long, J. R. Electronic Conductivity, Ferrimagnetic Ordering, and Reductive Insertion Mediated by Organic Mixed-Valence in a Ferric Semiquinoid Metal-Organic Framework. J. Am. Chem. Soc. 2015, 137, 15703-15711. 